\title{
RADICALS AND SUBDIRECT DECOMPOSITIONS OF $\Omega$-GROUPS
}

\author{
R. MLITZ AND S. VELDSMAN
}

(Received 20 May 1988; revised 25 June 1988)

Communicated by B. J. Gardner

\begin{abstract}
Starting with a class $\mathscr{H}$ of $\Omega$-groups, necessary and sufficient conditions on $\mathscr{H}$ are given to ensure that the corresponding Hoehnke radical $\rho$ (determined by the subdirect closure of $\mathscr{H}$ semisimple class) is a radical in the sense of Kurosh and Amitsur; has a hereditary semisimple class; satisfies the ADS-property; has a hereditary radical class or satisfies $\rho N \cap I \subseteq \rho I$ and lastly, have both a hereditary radical and semisimple class or satisfies $\rho N \cap I=\rho I$.
\end{abstract}

1980 Mathematics subject classification (Amer. Math. Soc.) (1985 Revision): 08 A 05, 16 A 21.

\section{Introduction}

Radicals are an important tool in structure theory since they yield subdirect decompositions of the semisimple algebras. Vice versa, it is often interesting to start with concrete class $\mathscr{H}$ of algebras and to consider the radical whose semisimple class is the subdirect closure of $\mathscr{M}$. Such a radical is always a radical in the sense of Hoehnke [6], but in general it will not satisfy further conditions, in particular, it need not be a radical in the classical Kurosh-Amitsur sense. Thus there is the problem of finding a correspondence between properties of the radical and of the class $\mathscr{M}$. Investigations of this kind have been done by Rashid and Wiegandt [17] and by Anderson, Kaarli and Wiegandt [1].

(C) 1990 Australian Mathematical Society $0263-6115 / 90 \$ A 2.00+0.00$ 
In this note we continue these investigations, giving necessary and sufficient conditions on $\mathscr{M}$ to ensure that the corresponding radical $\rho$

(1) is a radical in the sense of Kurosh and Amitsur (Section 2)

(2) has a hereditary semisimple class or satisfies the ADS-property (Section 3)

(3) has a hereditary radical class or satisfies $\rho N \cap I \subseteq \rho I$ for al $I \triangleleft N$ (Section 4)

(4) has both a hereditary radical and semisimple class or satisfies $\rho N \cap I=$ $\rho I$ for all $I \triangleleft N$ (Section 5 ).

In Section 6 we give several examples to show the independence of various conditions under discussion. Although we will work in a universal class of $\Omega$-groups, most of the results will remain true in any category with a zero-object and in which the appropriate variants of the homomorphism and isomorphism theorems of algebra are available.

For the basics on Hoehnke radicals, we refer to Hoehnke [6] and for Kurosh-Amitsur radicals, Divinsky [4] or Wiegandt [23] can be consulted.

\section{Preliminaries}

Let $\mathscr{A}$ be a universal class of $\Omega$-groups (that is, a class closed under taking homomorphic images and ideals); all considerations will be in $\mathscr{A}$. As usual, we denote by 0 the one-element $\Omega$-group in $\mathscr{A}$ and by $I \triangleleft N$ the fact that $I$ is an ideal of $N$. Essential ideals (that is, ideals $I$ satisfying $J \triangleleft N$ and $I \cap J=0$ implies $J=0$ ) are denoted by $I \triangleleft \circ N$. All subclasses of $\mathscr{A}$ under discussion will be assumed to be abstract, that is, containing 0 and closed under isomorphic copies. For a subclass $\mathscr{M}$ of $\mathscr{A}$, we denote by $\overline{\mathscr{K}}$ the subdirect closure of $\mathscr{M}$ in $\mathscr{A}$, that is,

$$
\overline{\mathscr{K}}:=\{N \in \mathscr{A} \mid N \text { is a subdirect sum of } \Omega \text {-groups from } \mathscr{K}\} \text {. }
$$

We call $\mathscr{M}$ subdirectly closed if $\mathscr{M}=\overline{\mathscr{M}}$. With an $N \in \mathscr{A}$, we associate two ideals, depending on $\mathscr{M}$, which are defined by

$$
\mathscr{K}(N):=\sum(I \triangleleft N \mid I \in \mathscr{M}) \text { and }(N) \mathscr{M}:=\bigcap(I \triangleleft N \mid N / I \in \mathscr{M}) \text {. }
$$

Note that $(N) \mathscr{M}=(N) \overline{\mathscr{K}}$ for all $N \in \mathscr{A}$. As usual, $\mathscr{U}$ and $\mathscr{S}$ will denote the upper radical and upper semisimple operators on a class $\mathscr{M} \subseteq \mathscr{A}$, that is,

$\mathscr{U} \mathscr{K}:=\{N \in \mathscr{A} \mid N$ has no non-zero homomorphic image in $\mathscr{M}\}$ and

$\mathscr{S} \mathscr{M}:=\{N \in \mathscr{A} \mid N$ has no non-zero ideals which are in $\mathscr{M}\}$.

Later it will be useful to remember that $\mathscr{U} \mathscr{M}=\mathscr{U} \overline{\mathscr{M}}$ for any $M \subseteq \mathscr{A}$. 
A subclass of $\mathscr{A}$ is hereditary (homomorphically closed) if it is closed under the taking of ideals (homomorphic images respectively).

A Hoehnke radical ( $H$-radical for short) [6] on $\mathscr{A}$ is a mapping $\rho$ assigning to each $N \in \mathscr{A}$ an ideal $\rho N$ of $N$ and satisfying

( $\rho 1) f(\rho N) \subseteq \rho(f(N))$ for any homomorphism $f$ defined on $N \in \mathscr{A}$,

( $\rho 2) \rho(N / \rho N)=0$ for all $N \in \mathscr{A}$.

With each $H$-radical $\rho$ we associate two subclasses of $\mathscr{A}$, namely:

$$
\mathscr{S}_{\rho}:=\{N \in \mathscr{A} \mid \rho N=0\} \text { and } \mathscr{R}_{\rho}:=\{N \in \mathscr{A} \mid \rho N=N\},
$$

called the semisimple class and the radical class of $\rho$ respectively. From [6] we know that the radical classes are exactly the homomorphically closed subclasses of $\mathscr{A}$ and the semisimple classes exactly the subdirectly closed subclasses of $\mathscr{A}$. Any $H$-radical $\rho$ is uniquely determined by $\mathscr{S}_{\rho}$ via $\rho N=$ $(N) \mathscr{S}_{\rho}$. It follows that for an arbitrary subclass $\mathscr{M}$ of $\mathscr{A}$, the mapping $\rho$ defined by $\rho N:=(N) \mathscr{M}=(N) \overline{\mathscr{K}}$ is always an $H$-radical with $\mathscr{S}_{\rho}=\overline{\mathscr{K}}$. In the sequel it will be called the $H$-radical corresponding to $\mathscr{M}$; it is the largest $H$-radical for which all $\Omega$-groups in $\mathscr{H}$ are semisimple, that is, the upper $H$ radical determined $\mathscr{M}$. For this radical, we have $\mathscr{R}_{\rho}=\mathscr{U} \mathscr{S}_{\rho}=\mathscr{U} \overline{\mathscr{M}}=\mathscr{U} \mathscr{M}$.

For any $\mathscr{K} \subseteq \mathscr{A}$, the class $\mathscr{U} \mathscr{M}$ is always homomorphically closed; hence $\mathscr{S} \mathscr{U} \mathscr{K}$ is subdirectly closed. Consequently there are two $H$-radicals $\rho$ and $\rho^{\prime}$, not necessarily comparable in any way, with $\mathscr{S}_{\rho}=\overline{\mathscr{M}}$ and $\mathscr{S}_{\rho^{\prime}}=\mathscr{S} \mathscr{U} \mathscr{K}$.

In connection with the equality $\rho N=(N) \mathscr{M}$, we recall the concept of an $\mathscr{M}$-representable ideal: Let $\mathscr{M} \subseteq \mathscr{A}, N \in \mathscr{A}$. An ideal $I \triangleleft N$ is $\mathscr{M}$ representable if $I$ is of the form $I=\bigcap_{\alpha} L_{\alpha}$ where each $L_{\alpha} \triangleleft N$ and satisfies $N / L_{\alpha} \in \mathscr{M}$.

Such ideals will be denoted by $I \triangleleft N$. Each $N \in \mathscr{A}$ always contains a smallest $\mathscr{M}$-representable ideal, namely $(N) \mathscr{M}$. If $\mathscr{M} \subseteq \mathscr{A}$ is homomorphically closed, we may define an $H$-radical $\rho$ by

$$
\rho N= \begin{cases}N & \text { if } N \in \mathscr{M}, \\ 0 & \text { if } N \notin \mathscr{M} .\end{cases}
$$

By construction, this $H$-radical is the smallest one for which all $\Omega$-groups from $\mathscr{H}$ are radical, that is the lower $H$-radical determined by $\mathscr{M}$. In general there are several different $H$-radicals with the same radical class (compare [6]). To save a lot of unnecessary formulations, we also mention the following:

1.1 Theorem. If $\mathscr{M} \subseteq \mathscr{A}, \rho$ the corresponding $H$-radical and we have a Proposition: $\rho$ satisfies some condition ( $x$ ) if and only if $\mathscr{M}$ satisfies some condition $(y)$, then there always is a corresponding 
Corollary: $\rho$ satisfies condition $(x)$ if and only if any $K \subseteq \mathscr{A}$ with $\overline{\mathscr{K}}=\overline{\mathscr{K}}$ satisfies condition $(y)$.

Moreover, such a condition (y) is always subdirectly invariant (that is a subclass of $\mathscr{A}$ satisfies condition (y) if and only if its subdirect closure in $\mathscr{A}$ satisfies condition $(y)$ ).

Proof. Let $\mathscr{K} \subseteq \mathscr{A}$ with $\overline{\mathscr{K}}=\overline{\mathscr{M}}$. Let $\rho^{\prime}$ be the $H$-radical defined by $\rho^{\prime} N:=(N) \mathscr{K}$. Then for all $N \in \mathscr{A}, \rho^{\prime} N=(N) \mathscr{K}=(N) \overline{\mathscr{K}}=(N) \overline{\mathscr{K}}=$ $(N) \mathscr{M}=\rho N$, that is $\rho^{\prime}=\rho$. Thus, $\rho$ satisfies $(x)$ if and only if $\rho^{\prime}$ satisfies $(x)$ if and only if $\mathscr{K}$ satisfies $(y)$ by the Proposition. The last assertion can easily be verified.

As an application of this result, we have

1.2 Proposition. Let $\mathscr{M} \subseteq \mathscr{A}, \rho$ the corresponding $H$-radical. Then there is no condition ( $x$ ) such that $\rho$ satisfies condition $(x)$ if and only if $\mathscr{M}$ is hereditary.

Proof. In view of Theorem $1.1 \mathrm{it}$ is sufficient to show that hereditariness is not subdirectly invariant. Although $\mathscr{M} \subseteq \mathscr{A}$ hereditary implies $\overline{\mathscr{M}}$ hereditary (compare Proposition 3 in [1]), the converse is not true. Let $\mathscr{A}$ be the class of all associative rings. Let $B$ be a non-zero subdirectly reducible ring and let $\mathscr{K}$ be the class of all subdirectly irreducible rings together with $\mathscr{D}(B)$, the Dorroh extension of $B$. Then $\overline{\mathscr{H}}=\mathscr{A}$ (by the well-known Birkhoff theorem) which is clearly hereditary, but $\mathscr{M}$ is not hereditary.

\section{Kurosh-Amitsur radicals}

A subclass $\mathscr{R} \subseteq \mathscr{A}$ is a Kurosh-Amitsur radical class (KA-radical class for short) if $\mathscr{R}$ is homomorphically closed and for all $N \in \mathscr{A}, \mathscr{R}(N) \in \mathscr{R}$ and $\mathscr{R}(N / \mathscr{R}(N))=0$.

Any KA-radical class $\mathscr{R}$ determines an $H$-radical $\rho$ by defining $\rho N:=$ $\mathscr{R}(N)$ for all $N \in \mathscr{A}$. In such a case, $\mathscr{R}_{\rho}=\mathscr{R}$ and $\mathscr{S}_{p}=\mathscr{P} \mathscr{R}$. In [15] it was shown that if $\rho$ is an $H$-radical, then $\mathscr{R}_{\rho}$ is a KA-radical class with $\mathscr{R}_{\rho}(N)=\rho N$ for all $N \in \mathscr{A}$ if and only if $\rho$ satisfies

( $\rho 3) \rho \rho N=\rho N$ for all $N \in \mathscr{A}$ (idempotence), and

( $\rho 4) \rho I=I \triangleleft N \in \mathscr{A}$ implies $I \subseteq \rho N$ (completeness).

Consequently, $H$-radicals which are idempotent and complete are called $K A$ radicals. Conditions $(\rho 3)$ and $(\rho 4)$ are independent; this follows from Examples 6.1 and 6.2 . 
A subclass $\mathscr{M} \subseteq \mathscr{A}$ is regular if $0 \neq I \triangleleft N \in \mathscr{M}$ implies the existence of an ideal $K \triangleleft I$ such that $0 \neq I / K \in \mathscr{M} . \mathscr{M}$ is an r-class if every $0 \neq N \in \mathscr{M}$ has an image $0 \neq N / I \in \mathscr{S O U}_{\mathscr{M}}$. Clearly any hereditary class is regular and every regular class is an $r$-class. Moreover, $\mathscr{S}_{p}$ is regular for any KA-radical $\rho$. Not every $r$-class is regular (see, for example, Leavitt [10]).

2.1 Proposition. Let $\mathscr{M} \subseteq \mathscr{A}, \rho$ the $H$-radical corresponding $\mathscr{M}$. Then the following are equivalent:

(1) $\mathscr{M}$ is an r-class,

(2) $\mathscr{R}_{\rho}(=\mathscr{U} \mathscr{M})$ is a KA-radical class,

(3) (a) $\mathscr{R}_{\rho}(N) \in \mathscr{R}_{\rho}$ for all $N \in \mathscr{A}$,

(b) $\mathscr{R}_{\rho}$ is closed under extensions (that is if $I \triangleleft N$ with $I$ and $N / I$ both in $\mathscr{R}_{\rho}$, then $N \in \mathscr{R}_{\rho}$ ).

The above equivalences are well-known; so are those in the next result (in any case, they can easily be verified):

2.2 Proposition. Let $\mathscr{M} \subseteq \mathscr{A}$ and let $\rho$ be the corresponding $H$-radical. The following are equivalent:

(1) $\mathscr{M}$ is regular,

(2) $\mathscr{M} \subseteq \mathscr{S} \mathscr{U} \mathscr{M}$,

(3) $\rho$ is complete,

(4) $\mathscr{R}_{\rho}(N) \subseteq \rho N$ for all $N \in \mathscr{A}$.

2.3 Remark. If $\mathscr{M}$ is not an $r$-class, then (even if $\rho$ is idempotent) we may have $0 \neq \mathscr{R}_{p}(N) \in \mathscr{M}$ for some $N$ as can be verified from Examples 6.3 and 6.4. The idempotence of $\rho$ always implies the dual of (4) in Proposition 2.2 namely $\left(4^{*}\right): \rho N \subseteq \mathscr{R}_{\rho}(N)$ for all $N \in \mathscr{A}$. If $\mathscr{M}$ is regular, then $\left(4^{*}\right)$ holds iff $\rho N=\mathscr{R}_{\rho}(N)$ for all $N \in \mathscr{A}$ iff $\rho$ is a KA-radical iff $\rho$ is idempotent (compare [15]). Example 6.3 shows that $\left(4^{*}\right)$ does not imply idempotence, that is there exists an $H$-radical satisfying $\left(4^{*}\right)$ but neither $(\rho 3)$ nor $(\rho 4)$.

A straightforward checking yields

2.4 Proposition. Let $\mathscr{M} \subseteq \mathscr{A}, \rho$ the H-radical corresponding to $\mathscr{M}$. Then $\rho$ is idempotent if and only if $\mathscr{M}$ fulfills

(T) $I \triangleleft N \in \mathscr{A}$ with $(I) \mathscr{M} \neq I$ implies $I \neq(N) \mathscr{M}$ (in fact, $(N) \mathscr{K} \subsetneq I)$.

Note that condition (T) can be reformulated by

$I \triangleleft N \in \mathscr{A}$ and $K \triangleleft I$ with $0 \neq I / K \in \mathscr{M}$ implies the existence of an ideal $J \triangleleft N$ such that $0 \neq N / J \in \mathscr{M}$ and $I \not \subset J$. 
Thus, (T) is a weaker form of condition (S) for $\mathscr{N}=\mathscr{M}$ used by Leavitt and Van Leeuwen [14]—we claim the property for $\mathscr{M}$-representable ideals; they for arbitrary ideals.

2.5 Corollary. Let $\mathscr{M} \subseteq \mathscr{A}$, $\rho$ the corresponding $H$-radical. The following are equivalent:

(1) $\rho$ is a KA-radical,

(2) $\overline{\mathscr{M}}=\mathscr{S} \mathscr{R}$ for a $K A$-radical class $\mathscr{R}$,

(3) $\mathscr{M}$ is regular and fulfils condition $(\mathrm{T})$,

(4) $\mathscr{R}_{\rho}(N)=\rho N$ for all $N \in \mathscr{A}$.

It is well-known that if $\mathscr{M}$ is a regular subclass of $\mathscr{A}$, then $\mathscr{U} \mathscr{M}$ is a KAradical class and $\mathscr{S} \mathscr{U} \mathscr{H}$ is the smallest KA-semisimple class which contains $\mathscr{M}$; hence $\mathscr{M} \subseteq \overline{\mathscr{M}} \subseteq \mathscr{S} \mathscr{U} \mathscr{M}$. In this case, if $\rho$ is the $H$-radical corresponding to $\mathscr{M}$, then $\mathscr{R}_{\rho}=\mathscr{U} \mathscr{K}$ is a KA-radical class, but in general the inclusion $\mathscr{R}_{\rho}(N) \subseteq \rho N$ is strict. However, imposing condition $(\mathrm{T})$ on $\mathscr{M}$ ensures that $\overline{\mathscr{H}}=\mathscr{S} \mathscr{U} \mathscr{M}$. This was the approach of Rashid and Wiegandt [17] who first made use of condition $(\mathrm{T})$.

\section{Heredity properties connected with semisimple classes}

The hereditariness of the corresponding radical or semisimple class may provide important information about the radical. We start our investigations with heredity properties linked with the semisimple class.

3.1 Proposition. Let $\mathscr{M} \subseteq \mathscr{A}, \rho$ the corresponding $H$-radical. Then the following are equivalent:

(1) $\rho$ is s-hereditary (that is $\rho I \subseteq \rho N \cap I$ for all $I \triangleleft N \in \mathscr{A}$ );

(2) $\mathscr{S}_{\rho}(=\overline{\mathscr{M}})$ is hereditary;

(3) $I \triangleleft N \in \mathscr{M}$ implies $I \in \overline{\mathscr{M}}$.

Proof. That (1) implies (2) implies (3) is straightforward. To show that (3) implies (1), consider $J \triangleleft N \in \mathscr{A}$ with $N / J \in \mathscr{M}$. For any $I \triangleleft N$, we have $I /(I \cap J) \cong(I+J) / J \triangleleft N / J \in \mathscr{M}$ and in view of (3), it follows that $I /(I \cap J) \in \mathscr{\mathscr { H }}$. Hence $\rho I \subseteq J \cap I$ and by the definition of $\rho$ the validity of (1) follows.

Any one of the conditions (1) to (3) above, trivially implies ( $\rho 4)$; the converse is not true since there are KA-radicals with non-hereditary semi-simple classes (see for example Leavitt and Armendariz [13] or our Example 6.5). 
Example 6.6 shows that none of the conditions (1) to (3) implies idempotence.

In the classical KA-radical theory of associative rings, the hereditariness of the semisimple class is ensured by the Anderson-Divinsky-Sulinski-property (ADS-property): An $H$-radical $\rho$ has the $A D S$-property if $I \triangleleft N \in \mathscr{A}$ implies $\rho I \triangleleft N$. As we shall see later, for $H$-radicals $\rho$ the ADS-property is not in relation with the hereditariness of $\mathscr{S}_{\rho}$. For a characterization of the radicals with the ADS-property, the next two conditions on a class $\mathscr{M} \subseteq \mathscr{A}$ are important.

(G) $K \triangleleft I \triangleleft N \in \mathscr{A}$ with $I / K \in \mathscr{M}$ implies the existence of an ideal $B \triangleleft N$ such that $B \subseteq K$ and $I / B \in \mathscr{M}$.

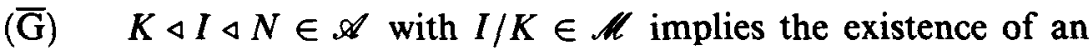
ideal $B \triangleleft N$ such that $B \subseteq K$ and $I / B \in \overline{\mathscr{M}}$.

The first one $(\mathrm{G})$ is equivalent to a condition introduced by Rjabuhin [18] to characterize ideal-hereditary radicals-see condition (A) in Buys [3], Definition 2.1 .

A further condition on a class $\mathscr{M}$ which is of relevance here is

$$
K \triangleleft I \triangleleft N \in \mathscr{A} \text { and } I / K \in \mathscr{M} \text { implies } K \triangleleft N \text {. }
$$

Clearly $(F)$ implies $(G)$ implies $(\bar{G})$. In general, these implications cannot be reversed: first we need

3.2 Proposition. Let $\mathscr{M} \subseteq \mathscr{A}, \rho$ the corresponding $H$-radical. Then the following are equivalent:

(1) $\rho$ has the ADS-property;

(2) $\mathscr{M}$ satisfies condition $(\overline{\mathrm{G}})$;

(3) $\overline{\mathscr{M}}$ satisfies condition $(\mathrm{G})$.

Proof. If $\rho$ has the ADS-property, then $\rho I \triangleleft N$ for all $I \triangleleft N \in \mathscr{A}$. Hence, if $K \triangleleft I \triangleleft N \in \mathscr{A}$ with $I / K \in \mathscr{K}$, then $(\overline{\mathrm{G}})$ is fulfilled with $B:=\rho I$; hence (1) implies (2). Suppose $\mathscr{M}$ satisfies condition $(\overline{\mathrm{G}})$. We now show that $\overline{\mathscr{K}}$ fulfils (G). Let $K \triangleleft I \triangleleft N \in \mathscr{A}$ with $I / K \in \overline{\mathscr{M}}$. Hence $I / K$ is of the form

$$
I / K \cong \sum_{\text {subd }}\left(I / K_{\alpha} \mid K_{\alpha} \triangleleft I \text { such that } I / K_{\alpha} \in \mathscr{M} \text { and } K=\bigcap_{\alpha} K_{\alpha}\right) \text {. }
$$

Since $\mathscr{M}$ satisfies $(\overline{\mathrm{G}})$, for each $\alpha$ there is an ideal $B_{\alpha} \triangleleft N$ such that $B_{\alpha} \subseteq K_{\alpha}$ and $I / B_{\alpha} \in \overline{\mathscr{M}}$. Let $B=\bigcap_{\alpha} B_{\alpha}$. Then $B \triangleleft N, B \subseteq \bigcap_{\alpha} K_{\alpha}=K$ and

$$
I / B \cong \sum_{\text {subd }}\left(I / B_{\alpha}\right) \in \overline{\overline{\mathscr{H}}}=\overline{\mathscr{K}} \text {. }
$$


Lastly we show that (3) implies (1). Let $I \triangleleft N \in \mathscr{A}$. Since $I / \rho I \in \mathscr{S}_{\rho}=\overline{\mathscr{M}}$, condition (G) on $\overline{\mathscr{K}}$ implies the existence of an ideal $B \triangleleft N$ with $B \subseteq \rho I$ and $I / B \in \overline{\mathscr{K}}$. The latter property yields $\rho I \subseteq B$; hence $\rho I=B \triangleleft N$.

It is now easy to see that $(F)$ is in general stronger than $(G)$. If $\mathscr{A}$ is the class of all associative rings, then every KA-radical $\rho$ has the ADS-property, hence $\mathscr{S}_{\rho}$ fulfils condition (G). In [1, Theorem 2], it was shown that every regular class which satisfies condition (F) must consist of semiprime rings. This means that the semisimple class of any subidempotent radical (that is if $\rho A=A$ then $A^{2}=A$ ) satisfies (G) but not (F). By Theorem 1.1 and Proposition 3.2 it follows that $(\overline{\mathrm{G}})$ is subdirectly invariant; thus to show that $(G)$ is in general stronger than $(\bar{G})$ it is sufficient to show that $(G)$ is not subdirectly invariant (although it is retained under subdirect closures). But this follows from Example 6.7.

Using Proposition 3.2 and Corollary 2.5, we have

3.3 Corollary. For $M \subseteq \mathscr{A}$, the $H$-radical corresponding to $\mathscr{M}$ is a $K A$-radical with the ADS-property if and only if $\mathscr{M}$ is regular and satisfies conditions $(\mathrm{T})$ and $(\overline{\mathrm{G}})$.

We call a class $\mathscr{M} \subseteq \mathscr{A}$ closed under essential $\mathscr{M}$-representable extensions (for short CE $\mathscr{K}$ E-class) if $I \triangleleft N \in \mathscr{A}$ and $I \triangleleft \circ N$, then $I \in \mathscr{K}$ implies $N \in \mathscr{K}$.

3.4 Proposition. If $\mathscr{M} \subseteq \mathscr{A}$ is a CE $\mathscr{M}$ E-class, regular and fulfils (G), then the corresponding $H$-radical is a KA-radical and has the ADS-property.

Proof. Let $\rho$ be the $H$-radical corresponding to $\mathscr{M}$. We only have to show that $\rho$ is idempotent. Let $N \in \mathscr{A}$. If $\rho \rho N \neq \rho N$, then there exists an ideal $K \triangleleft \rho N$ such that $0 \neq \rho N / K \in \mathscr{M}$. By condition (G), there is an ideal $B \triangleleft N$ with $B \subseteq K$ and $\rho N / B \in \mathscr{M}$. Since $\rho N$ is an $\mathscr{M}$-representable ideal of $N$, $\rho N / B$ is an $\mathscr{M}$-representable ideal of $N / B$. Indeed, if $\rho N=\bigcap_{\alpha} L_{\alpha}$ where $L_{\alpha} \triangleleft N$ with $N / L_{\alpha} \in \mathscr{M}$, then $B \subseteq L_{\alpha}$ for each $\alpha$. Hence,

$$
\rho N / B=\bigcap_{\alpha}\left(L_{\alpha} / B \mid L_{\alpha} / B \triangleleft N / B \text { and }(N / B) /\left(L_{\alpha} / B\right) \cong N / L_{\alpha} \in \mathscr{K}\right) \text {. }
$$

It is well-known that if $C / B \triangleleft N / B$ is maximal with respect to $(\rho N / B) \cap$ $(C / B)=0$, then $\rho N / B \cong(\rho N+C) / C \triangleleft \circ N / C$. Since $\mathscr{H}$ is a CE $\mathscr{K}$ E-class, $N / C \in \mathscr{M}$ follows. This means $\rho N \subseteq C$ which contradicts $\rho N / B \neq 0$. Thus $\rho \rho N=\rho N$.

Since $(G)$ is in general stronger than $(\bar{G})$, the converse of the above result is not true (compare Example 6.7). Notice that $(\mathrm{G})$ and heredity of $\mathscr{M}$ do not imply that $\mathscr{M}$ is a CE $\mathscr{M}$ E-class (compare Example 6.6). 
3.5 CoRollary. $\mathscr{H} \subseteq \mathscr{A}$ is the semisimple class of a KA-radical with the $A D S$-property if and only if $\mathscr{M}$ is regular, closed under subdirect sums, satisfies condition $(\mathrm{G})$ and is a CE $\mathscr{M}$ E-class.

Proof. We only have to verify that if $\mathscr{M}=\mathscr{S}_{\rho}$ for a KA-radical $\rho$ with the ADS-property, then $\mathscr{M}$ satisfies condition $(\mathrm{G})$ and is an CE $\mathscr{K}$ E-class. The validity of $(\mathrm{G})$ follows from Proposition 3.2. Consider thus $I \triangleleft \circ N \in \mathscr{A}$ with $I=\bigcap_{\alpha} L_{\alpha} \in \mathscr{M}$ where $L_{\alpha} \triangleleft N$ with $N / L_{\alpha} \in \mathscr{M}$. By the definition of $\rho$, $\rho N \subseteq I$. Since $\rho$ is a KA-radical, $\rho N \subseteq \rho I=0$; hence $N \in \mathscr{S}_{\rho}=\mathscr{M}$.

3.6 CoRollary. Let $\mathscr{M} \subseteq \mathscr{A}$. Then the $H$-radical corresponding to $\mathscr{M}$ is a KA-radical with the ADS-property if and only if $\mathscr{M}$ is regular, fulfils $(\bar{G})$ and $\overline{\mathscr{M}}$ is a CE $\mathscr{M}$ E-class.

As mentioned earlier, it is well-known that for KA-radicals $\rho$ (that is under the presence of $(\rho 3)$ and $(\rho 4))$, the ADS-property implies the hereditariness of $\mathscr{S}_{\rho}$. Since there are non-hereditary semisimple classes of KA-radicals, ( $\left.\rho 3\right)$ and $(\rho 4)$ imply neither $s$-heredity nor the ADS-property (compare [13]). Let us mention that there are quite a few natural universal classes where $(\rho 3)$ and ( $\rho 4$ ) do imply the ADS-for example, the associative rings, alternative rings, abelian groups and the class of all groups. However, Examples 6.1 and 6.2 show that $(\rho 3)$ and the ADS-property do not imply $(\rho 4)$ (and consequently $\mathscr{S}_{\rho}$ is not hereditary) and that the ADS-property and ( $\left.\rho 4\right)$ do not imply ( $\left.\rho 3\right)$ nor the hereditariness of $\mathscr{S}_{\rho}$. In view of these examples, one might ask for conditions which, under the presence of the ADS-property, ensure that the semisimple class is hereditary. The next result provides some information.

3.7 Proposition. Let $\rho$ be an $H$-radical with the ADS-property. Then $\rho$ is $s$-hereditary if and only if $\rho$ is complete and hereditarily idempotent on $\mathscr{S}_{\rho}$ (that is $I \triangleleft N \in \mathscr{S}_{\rho}$ implies $\rho \rho I=\rho I$ ).

Proof. Any $s$-hereditary $H$-radical satisfies, without the assumption of ADS, the two conditions. Conversely, if $I \triangleleft N \in \mathscr{S}_{\rho}$, then $\rho \rho I=\rho I$ and by the ADS-property and the completeness of $\rho$, we have $\rho I \subseteq \rho N=0$, that is $I \in \mathscr{S}_{\rho}$.

\section{Heredity properties linked with radical classes}

For heredity properties linked with $\mathscr{R}_{\rho}$, the situation is more complicated than for those linked with $\mathscr{S}_{\rho}$. It is well-known that for KA-radicals $\rho$ the 
properties of being r-hereditary (that is $\rho N \cap I \subseteq \rho I$ for all $I \triangleleft N \in \mathscr{A}$ ) and of having a hereditary radical class $\mathscr{R}_{\rho}$ are equivalent. Note that for an $r$-hereditary $H$-radical $\rho, \rho$ is always idempotent and $\mathscr{R}_{\rho}$ is hereditary. However, the converse is not true in view of the next two propositions.

4.1 Proposition. For an H-radical $\rho$, conditions (1) and (2) below are equivalent:

(1) $\rho$ is $r$-hereditary;

(2) (a) $\rho$ is idempotent,

(b) $\mathscr{R}$ is hereditary,

(c) $\rho(\rho N \cap I) \subseteq \rho I$ for all $I \triangleleft N \in \mathscr{A}$.

Proof. That (1) implies (2) is straightforward. Suppose (2) holds and take $I \triangleleft N \in \mathscr{A}$. Then $\rho N \cap I \triangleleft \rho N \in \mathscr{R} \rho$ implies $\rho N \cap I=\rho(\rho N \cap I) \subseteq \rho I$ by (a), (b) and (c). Hence $\rho$ is $r$-hereditary.

Note that if $\rho$ is complete, then (a) and (b) imply (c); the radical then being a KA-radical. If $\rho$ is a KA radical for which either $\mathscr{R}_{\rho}$ or $\mathscr{S}_{\rho}$ is hereditary, then $\rho$ satisfies (c), but (c) does not imply the hereditariness of either $\mathscr{R}_{\rho}$ or $\mathscr{S}_{p}$ (compare Example 6.5). Further, Example 6.11 shows that not every KA-radical has to satisfy condition (c). Remark also that, in view of Example 6.1, $r$-hereditary and ADS do not imply completeness.

4.2 Proposition. For $H$-radicals on $\mathscr{A}$, conditions (a), (b) and (c) are independent.

Proof. We show that these conditions are in fact independent in a very strong sense by giving a model for each of the following systems (where $\overline{(x)}$ is the negation of $(x), x \in\{a, b, c\})$ :

(1) $(a),(b), \overline{(c)}$-Example 6.4,

(2) $(a), \overline{(b)},(c)$-Example 6.5,

(3) $\overline{(a)},(b),(c)$,

(4) $\overline{(a)}, \overline{(b)}, \overline{(c)}$-Example 6.8,

(5) $\overline{(a)}, \overline{(b)},(c)$-Example 6.10,

(6) $\overline{(a)},(b), \overline{(c)}$-Example 6.9,

(7) $(a), \overline{(b)}, \overline{(c)}$-Example 6.11 .

Concerning (3), note the following. Let $\mathscr{M}$ be a subclass of $\mathscr{A}$ for which both $\mathscr{M}$ and $\mathscr{U} \mathscr{M}$ are hereditary and $\mathscr{M}$ is a proper subclass of $\mathscr{S} \mathscr{U} \mathscr{M}$ (since $\mathscr{H}$ is regular, $\mathscr{K} \subseteq \bar{M} \subseteq \mathscr{S} \mathscr{U} \mathscr{M}$ ). If $\rho$ is the $H$-radical corresponding $\mathscr{M}$, then $\mathscr{S}_{\rho}=\overline{\mathscr{M}}$ and $\mathscr{R}_{\rho}=\mathscr{U} \mathscr{M}$ are both hereditary. Hence $\rho$ fulfils (b) and (c) but $\rho$ is not idempotent. Indeed, if $\rho$ were idempotent, then $\rho N \in \mathscr{R}_{p}$. Hence $\rho N \subseteq \mathscr{R}_{\rho}(N)=(N) \mathscr{S} \mathscr{R} \rho=(N) \mathscr{S} \mathscr{U} \mathscr{M}=(N) \mathscr{S} \mathscr{U} \overline{\mathscr{M}} \subseteq(N) \bar{M}=\rho N$, that 
is $\rho N=\mathscr{R}_{\rho}(N)$. Consequently $\overline{\mathscr{K}}=\mathscr{S} \mathscr{U} \mathscr{M}$ which contradicts our choice of $\mathscr{M}$. The existence of such classes $\mathscr{M}$ follows from Example 6.6 which completes the proof.

4.3 Remark. Let $\mathscr{M} \subseteq \mathscr{A}, \rho$ the corresponding $H$-radical. Since $\mathscr{R}_{\rho}=$ $\mathscr{U} \mathscr{M}=\mathscr{U} \overline{\mathscr{M}}$ and $N / \rho N \in \overline{\mathscr{M}}$ for all $N \in \mathscr{A}$, straightforward arguments show that the hereditariness of $\mathscr{R}_{\rho}$ is equivalent to any one of the following conditions.

(1) $K \triangleleft I \triangleleft N \in \mathscr{A}$ with $0 \neq I / K \in \mathscr{M}$ implies the existence of an ideal $J \triangleleft N$ such that $0 \neq N / J \in \mathscr{M}$.

(2) $K \triangleleft I \triangleleft N \in \mathscr{A}$ with $0 \neq I / K \in \mathscr{M}$ implies the existence of an ideal $J \triangleleft N$ such that $0 \neq N / J \in \overline{\mathscr{M}}$.

(3) $K \triangleleft I \triangleleft N \in \mathscr{A}$ with $0 \neq I / K \in \overline{\mathscr{M}}$ implies the existence of an ideal $J \triangleleft N$ such that $0 \neq N / J \in \mathscr{M}$.

(4) $K \triangleleft I \triangleleft N \in \mathscr{A}$ with $0 \neq I / K \in \overline{\mathscr{M}}$ implies the existence of an ideal $J \triangleleft N$ such that $0 \neq N / J \in \overline{\mathscr{M}}$.

(5) $I \triangleleft N \in \mathscr{A}$ with $I \neq(I) \mathscr{M}$ implies the existence of an ideal $J \triangleleft N$ such that $0 \neq N / J \in \mathscr{M}$.

(6) $I \triangleleft N \in \mathscr{A}$ with $I \neq(I) \mathscr{M}$ implies the existence of an ideal $J \triangleleft N$ such that $0 \neq N / J \in \overline{\mathscr{K}}$.

From the above it is clear that conditions (1) to (6) are equivalent. In view of Proposition 4.1, it seems natural to look for some condition to add to (1) to (6) in order to ensure that $\rho$ is $r$-hereditary. Considering the meaning of $r$-hereditariness, Theorem 2 and Corollary 3 in Leavitt and Van Leeuwen [14] and the characterization obtained by Mlitz and Oswald ([16, Theorem 3.5]the proof carries over for $\Omega$-groups), we get " $I \cap J \subseteq K$ " or " $I \nsubseteq J$ " as possible candidates for such supplementary conditions. Thus for $i=1,2,3,4,5,6$ let us denote by $\left(i^{\prime}\right)$ the condition obtained from (i) in Remark 4.3 by adding "... and $I \cap J \subseteq K$ " for $i=1,2,3$ and 4 and adding "... and $I \cap J \subseteq(I) \mathscr{M}$ " for $i=5,6$. Furthermore, by $\left(i^{\prime \prime}\right)$ we denote the condition obtained from (i) in Remark 4.3 by adding "... and $I \nsubseteq J$ " for $i=1,2, \ldots, 6$.

4.4 Proposition. Let $\mathscr{M} \subseteq \mathscr{A}, \rho$ the $H$-radical corresponding to $\mathscr{M}$. The following are equivalent.

(1) $\rho$ is idempotent and $\mathscr{R}_{p}$ is hereditary;

(2) satisfies condition ( $\left.\mathrm{i}^{\prime \prime}\right)$ for any $i \in\{1,2,3,4,5,6\}$.

Proof. Since the ideal $J$ given by the implications in $\left(i^{\prime \prime}\right)$ is independent of $K$, it is clear that the equivalences ( $\left.1^{\prime \prime}\right)$ if and only if $\left(3^{\prime \prime}\right)$ if and only if $\left(5^{\prime \prime}\right)$ and $\left(2^{\prime \prime}\right)$ if and only if $\left(4^{\prime \prime}\right)$ if and only if $\left(6^{\prime \prime}\right)$ hold. Furthermore, since $\mathscr{M} \subseteq \bar{M}$, also $\left(1^{\prime \prime}\right)$ implies $\left(2^{\prime \prime}\right)$. Suppose $\mathscr{M}$ satisfies condition $\left(2^{\prime \prime}\right)$. For 
$I \triangleleft N \in \mathscr{A}$ with $(I) \mathscr{M} \neq I$, there is an ideal $J \triangleleft N$ such that $0 \neq N / J \in \overline{\mathscr{M}}$ with $I \nsubseteq J$. Hence $N / J$ is of the form

$$
N / J \cong \sum_{\text {subd }}\left(N / J_{\alpha} \mid J_{\alpha} \triangleleft N \text { with } N / J_{\alpha} \in \mathscr{H} \text { and } J=\bigcap_{\alpha} J_{\alpha}\right) .
$$

Clearly there is an $\alpha_{0}$ such that $I \nsubseteq J_{\alpha_{0}}$ which shows the validity of ( $\left(1^{\prime \prime}\right)$. We have thus showed that the conditions $\left(1^{\prime \prime}\right)$ to $\left(6^{\prime \prime}\right)$ are all equivalent. If $\rho$ is idempotent and $\mathscr{R}_{\rho}$ is hereditary, then $\mathscr{M}$ satisfies condition (1"). Indeed, if $I \triangleleft N \in \mathscr{A}$ and $(I) \mathscr{M} \neq I$, then $I \notin \mathscr{R}_{\rho}$ and thus also $N \in \mathscr{R}_{\rho}$. Hence there is an ideal $J \triangleleft N$ such that $0 \neq N / J \in \mathscr{M}$. If $I \subseteq J$ for all such ideals $J$, then $I \subseteq \rho_{N}$. But the idempotence of $\rho$ and the hereditariness of $\mathscr{R}_{\rho}$ yields $I \in \mathscr{R}_{p}$ which contradicts $I \notin \mathscr{R}_{p}$. Consequently condition $\left(1^{\prime \prime}\right)$ is fulfilled. Conversely, assume condition $\left(1^{\prime \prime}\right)$ holds. Then, so does condition (1) and $\mathscr{R}_{\rho}$ is hereditary. If $\rho$ is not idempotent, then $\rho \rho N \neq \rho N$ for some $N \in \mathscr{A}$. Applying condition (1") for $I:=\rho N$, there is an ideal $J \triangleleft N$ such that $0 \neq N / J \in \mathscr{M}$ and $\rho N \nsubseteq J$. But $N / J \in \mathscr{M}$ implies $\rho N \subseteq J$; clearly a contradiction. Thus $\rho$ is idempotent and the proof is complete.

4.5 Corollary. Let $\mathscr{M} \subseteq \mathscr{A}$, $\rho$ the corresponding $H$-radical. Then $\rho$ is an r-hereditary KA-radical if and only if $\mathscr{M}$ is regular and satisfies condition $\left(1^{\prime \prime}\right)$.

It is clear that condition ( $\left.1^{\prime \prime}\right)$ implies condition (T)-the converse is not true since there are KA-radicals with non-hereditary radical classes. Also, $\left(1^{\prime \prime}\right)$ implies (1), but not conversely; see Example 6.6.

4.6 Proposition. Let $\mathscr{M} \subseteq \mathscr{A}$, $\rho$ the corresponding $H$-radical. Then the following implications hold:

$\left(3^{\prime}\right)$ if and only if $\left(5^{\prime}\right)$ implies $\left(1^{\prime}\right)$ implies $\left(2^{\prime}\right)$ if and only if $\left(6^{\prime}\right)$ if and only if $\left(4^{\prime}\right)$ if and only if $\rho$ is $r$-hereditary implies $\left(1^{\prime \prime}\right)$.

Proof. Since $I /(I) \mathscr{M} \in \overline{\mathscr{M}}$ and $(I) \mathscr{M}$ is the smallest ideal of $I$ with this property, the implications $\left(3^{\prime}\right)$ if and only if $\left(5^{\prime}\right)$ implies $\left(1^{\prime}\right)$ and $\left(6^{\prime}\right)$ if and only if $\left(4^{\prime}\right)$ are obvious. Moreover, $\left(1^{\prime}\right)$ implies $\left(2^{\prime}\right)$ trivially since $\mathscr{H} \subseteq \overline{\mathscr{M}}$. We now show that $\left(2^{\prime}\right)$ implies $\left(6^{\prime}\right)$. To every $K \triangleleft I \triangleleft N \in \mathscr{A}$ with $0 \neq I / K \in \mathscr{M}$, there is some $J \triangleleft N$ (depending on $K$ and thus denoted in the sequel by $J_{K}$ ) with $0 \neq N / J_{K} \in \overline{\mathscr{M}}$ and $I \cap J_{K} \subseteq K$ by $\left(2^{\prime}\right)$. Hence, taking the intersection over all $K \triangleleft I$ sending $I$ into $\mathscr{M}-\{0\}$, we obtain

$$
0 \neq N / \bigcap_{K} J_{k} \in \overline{\mathscr{M}} \text { and } I \cap\left(\bigcap_{K} J_{K}\right)=\bigcap_{K}\left(I \cap J_{K}\right) \subseteq \bigcap_{K} K=(I) \mathscr{M} \text {, }
$$


that is $\left(6^{\prime}\right)$ holds. Suppose now $\left(4^{\prime}\right)$ holds and let $I \triangleleft N$. If $\rho I=I$, then trivially $I \cap \rho N \subseteq \rho I$. Suppose that $I / \rho I \neq 0$. By $\left(4^{\prime}\right)$, there is an ideal $J \triangleleft N$ such that $0 \neq N / J \in \overline{\mathscr{M}}$ with $I \cap J \subseteq \rho I$. Since $\rho N \subseteq J$, we have $I \cap \rho N \subseteq \rho I$, that is $\rho$ is $r$-hereditary. Finally, suppose $\rho$ is $r$-hereditary. Then ( $\left.2^{\prime}\right)$ holds, for if $K \triangleleft I \triangleleft N \in \mathscr{A}$ with $0 \neq I / K \in \mathscr{M}$, then $J:=\rho N$ fulfils the requirements of $\left(2^{\prime}\right)$. The last implication follows from Propositions 4.1 and 4.4 .

Condition $\left(3^{\prime}\right)$ is quite strong in view of

4.7 Proposition. $\mathscr{M} \subseteq \mathscr{A}$ fulfils condition $\left(3^{\prime}\right)$ if and only if $\mathscr{M}=\overline{\mathscr{K}}$ and $\mathscr{M}$ fulfils condition $\left(2^{\prime}\right)$.

Proof. Suppose $\left(3^{\prime}\right)$ holds and let $0 \neq N \in \overline{\mathscr{K}}$. Apply condition $\left(3^{\prime}\right)$ with $I=N$. Then there is an ideal $J \triangleleft N$ such that $N / J \in \mathscr{M}$ and $I \cap J \subseteq \rho I$. This means $J=N \cap J \subseteq \rho N=0$; hence $N \in \mathscr{M}$. Thus $\mathscr{M}=\overline{\mathscr{H}}$ and $\left(2^{\prime}\right)$ follows from Proposition 4.6. The converse is obvious because it can easily be verified that if $\mathscr{M}$ is subdirectly closed, the conditions $\left(1^{\prime}\right)$ to $\left(6^{\prime}\right)$ on $\mathscr{K}$ are all equivalent.

Note that by Theorem 1.1 conditions $\left(2^{\prime}\right),\left(4^{\prime}\right)$ and $\left(6^{\prime}\right)$ are subdirectly invariant. In general, $\left(1^{\prime}\right)$ is weaker than $\left(3^{\prime}\right)$; see Example 6.12. For the relationship between $\left(1^{\prime}\right)$ and $\left(2^{\prime}\right)$, see the end of Section 4 . Example 6.4 shows that $\left(1^{\prime \prime}\right)$ does not imply $\left(2^{\prime}\right)$. Of course, if $\mathscr{K}$ is regular, then $\left(2^{\prime}\right)$ and $\left(2^{\prime \prime}\right)$ coincide on $\mathscr{K}$.

Since $\mathscr{S}_{\rho}$ is subdirectly closed for any $H$-radical $\rho$, we have

4.8 COROllary. An $\mathrm{H}$-radical $\rho$ is $r$-hereditary if and only if $\mathscr{S}_{\rho}$ fulfils any one (and hence all) of the conditions $\left(1^{\prime}\right)$ to $\left(6^{\prime}\right)$.

4.9 Corollary. $\mathscr{M} \subseteq \mathscr{A}$ is the semisimple class of an r-hereditary $K A$ radical if and only if $\mathscr{M}$ is regular and satisfies conditions ( $\left.3^{\prime}\right)$.

4.10 Corollary. Let $\mathscr{M} \subseteq \mathscr{A}$. Then $\overline{\mathscr{K}}$ is the semisimple class of an $r$-hereditary KA-radical if and only if $\mathscr{M}$ is regular and fulfils condition (2').

In Corollary 4.9, condition $\left(3^{\prime}\right)$ cannot be replaced by the weaker condition (1') since there are regular subclasses of $\mathscr{A}$ which fulfil (1') without being subdirectly closed-see Example 6.12.

A further condition which has frequently been used in connection with the hereditariness of $\mathscr{U} \mathscr{M}$ is the closedness of $\mathscr{K}$ under essential extensions (see for example [1], [5], [19]). For $\mathscr{M} \subseteq \mathscr{A}$, define the essential cover $\mathscr{M}_{k}$ of $\mathscr{M}$ in $\mathscr{A}$ by $\mathscr{M}_{k}:=\{N \in \mathscr{A} \mid$ there is an ideal $I \triangleleft \circ N$ with $I \in \mathscr{M}\}$. We say that 
is closed under essential extensions if $\mathscr{M}_{k}=\mathscr{M}$. We will also use condition $\left(\mathrm{E}^{\prime}\right)$ on a class $\mathscr{M} \subseteq \mathscr{A}$ :

$\left(\mathrm{E}^{\prime}\right) \quad$ If $I \triangleleft N \in \mathscr{A}$ and $I \neq(I) \mathscr{M}$, then there exist ideals $U, V$ of $N$ such that $U \subseteq V$ and $0 \neq V / U \in \mathscr{M}$.

This condition is weaker than condition (E) used by Leavitt in [10] and [11]. Condition ( $\left.E^{\prime}\right)$ is trivially fulfilled if the $\triangleleft$-relation is transitive on $\mathscr{A}$. Moreover, $(\mathrm{G})$ implies $\left(\mathrm{E}^{\prime}\right)$, the converse being false in view of Example 6.7.

Since the condition $(F)$ used by Leavitt and Van Leeuwen [14] implies $\left(E^{\prime}\right)$, the following result strengthens Theorem 4(1)-(3) of [14] and generalizes the corresponding result from KA-radicals of associative rings to $H$-radicals of $\Omega$-groups.

4.11 Proposition. Let $\mathscr{M} \subseteq \mathscr{A}, \rho$ the corresponding $H$-radical. Then the following are equivalent:

(1) $\mathscr{R}_{p}(=\mathscr{U} \mathscr{M})$ is hereditary;

(2) $\mathscr{U} \mathscr{M}=\mathscr{U} \mathscr{M}_{k}$ and $\mathscr{M}$ fulfils condition $\left(\mathrm{E}^{\prime}\right)$;

(3) $\mathscr{U} \mathscr{M} \cap \mathscr{M}_{k}=0$ and $\mathscr{M}$ fulfils condition $\left(\mathrm{E}^{\prime}\right)$.

Proof. (1) implies (2): Since $\mathscr{M} \subseteq \mathscr{M}_{k}, \mathscr{U} \mathscr{M}_{k} \subseteq \mathscr{U} \mathscr{M}$. Let $N \in \mathscr{U} \mathscr{M}$. If $N \notin \mathscr{U} \mathscr{M}_{k}$, then there is an ideal $I \triangleleft N$ with $0 \neq N / I \in \mathscr{M}_{k}$. By definition of $\mathscr{M}_{k}$, there is an essential ideal $J / I \triangleleft \circ N / I$ with $J / I \in \mathscr{M}$. Since $\mathscr{R}_{\rho}$ is homomorphically closed and hereditary,this means $J / I \in \mathscr{R}_{\rho}=\mathscr{U} \mathscr{K}$; a contradiction. Thus $\mathscr{U} \mathscr{M}=\mathscr{U} \mathscr{M}_{k}$. The hereditariness of $\mathscr{R}_{\rho}$ implies that (1) of Remark 4.3 is satisfied; hence $\left(\mathrm{E}^{\prime}\right)$ holds with $U=J$ and $V=N$. Trivially (2) implies (3). Suppose (3) holds. If $\mathscr{R}_{\rho}$ is not hereditary, then there is an ideal $I \triangleleft N \in \mathscr{R}_{\rho}$ with $I \notin \mathscr{R}_{\rho}$. By $\left(\mathrm{E}^{\prime}\right)$ there are ideals $U, V$ of $N$ with $U \subseteq V$ and $0 \neq V / U \in \mathscr{M}$. Let $C / U \triangleleft N / U$ be maximal with respect to $V / U \cap C / U=0$. Then we have $0 \neq V / U \cong(V+C) / C \triangleleft \circ N / C$. Hence $N / C \in \mathscr{M}_{k}$. But $N \in \mathscr{R}_{p}$ implies $N / C \in \mathscr{R}_{p}$; clearly not possible. Thus $\mathscr{R}_{\rho}$ is hereditary.

By Theorem 1.1, conditions (2) and (3) of 4.11 are subdirectly invariant; thus, remembering that $\mathscr{U} \mathscr{K}=\mathscr{U} \overline{\mathscr{M}}$, we get

4.12 COROLlaRY. $\mathscr{R}_{\rho}$ hereditary is equivalent both to

$(\overline{2}) \mathscr{U} \mathscr{M}=\mathscr{U}(\overline{\mathscr{M}})_{k}$ and $\overline{\mathscr{M}}$ satisfies $\left(\mathrm{E}^{\prime}\right)$ and to

(3) $\mathscr{U} \mathscr{M} \cap(\overline{\mathscr{M}})_{k}=0$ and $\overline{\mathscr{M}}$ satisfies $\left(\mathrm{E}^{\prime}\right)$.

Notice that, when considered separately, none of the conditions $\mathscr{U} \mathscr{K}=$ $\mathscr{U}_{k}, \mathscr{U} \mathscr{M} \cap \mathscr{K}_{k}=0$ or $\left(\mathrm{E}^{\prime}\right)$ are subdirectly invariant. The first two conditions carry over from $\overline{\mathscr{K}}$ to $\mathscr{M}$, but not vice versa (compare the ring $A$ in Example 
6.13); ( $\left.E^{\prime}\right)$ for $\mathscr{K}$ implies trivially $\left(E^{\prime}\right)$ for $\overline{\mathscr{K}}$, the converse being false in view of Example 6.16.

4.13 Corollary. $\mathscr{M}_{k} \subseteq \overline{\mathscr{M}}$ (or equivalently, $\overline{\mathscr{K}}_{k}=\overline{\mathscr{M}}$ ) and $\mathscr{M}$ satisfies $\left(\mathrm{E}^{\prime}\right)$ implies $\mathscr{R}_{\rho}$ is hereditary.

The converse is not true in view of Example 6.2. Note that by Leavitt's example [11, Theorem 4], given here in Section 6 as Example 6.13, one may have $\mathscr{M}_{k}=\mathscr{M}$ without $\mathscr{R}_{p}=\mathscr{U} \mathscr{M}$ being hereditary (compare also Example 6.16). Thus the condition ( $\left.E^{\prime}\right)$ cannot be dropped in the above results. But we can prove the following.

4.14 Proposition. Let $\mathscr{M} \subseteq \mathscr{A}, \rho$ the corresponding $H$-radical. If $\rho$ is r-hereditary, then $\overline{\mathscr{M}}$ is closed under essential extensions, in particular, $\mathscr{M}_{K} \subseteq$ $\overline{\mathscr{M}}=(\overline{\mathscr{M}})_{k}=\overline{\mathscr{M}}_{k}$.

Proof. Let $I \triangleleft \circ N \in \mathscr{A}$ with $I \in \overline{\mathscr{M}}$. Since $\rho$ is $r$-hereditary, $\rho N \cap I \subseteq$ $\rho I=0$; thus $\rho N=0$ and $N \in \overline{\mathscr{K}}$, showing $(\overline{\mathscr{M}})_{k} \subseteq \overline{\mathscr{K}}$. Now obviously $\mathscr{M}_{k} \subseteq \overline{\mathscr{K}}=(\overline{\mathscr{K}})_{k}=\overline{\mathscr{M}}_{k}$.

The converse is not true. $\mathscr{M}_{k} \subseteq \overline{\mathscr{M}}$ does not imply even the hereditariness of $\mathscr{R}_{\rho}$ (compare Example 6.13). Moreover, $r$ - and $s$-hereditary do not imply $\mathscr{M}_{k} \subseteq \mathscr{M}$ (compare Example 6.14). Next let us exhibit how the above results can be strengthened in the presence of the ADS-property, that is of $(\overline{\mathbf{G}})$ for $\mathscr{M}$. Since $\overline{\mathscr{M}}$ has $(\mathrm{G})$ if and only if $\mathscr{M}$ has $(\overline{\mathrm{G}})$ (compare with Proposition 3.2) and $(G)$ implies $\left(E^{\prime}\right)$, we first obtain from Corollary 4.12 lent:

4.15 Proposition. Let $\mathscr{M} \subseteq \mathscr{A}$ fulfil $(\overline{\mathrm{G}})$; then the following are equiva-

(1) $\mathscr{R}_{\rho}=\mathscr{U} \mathscr{M}$ is hereditary;

(2) $\mathscr{U} \mathscr{M}=\mathscr{U}(\overline{\mathscr{M}})_{k}$;

(3) $\mathscr{U} \mathscr{K} \cap(\overline{\mathscr{K}})_{k}=0$.

4.16 COROllary. Let $\mathscr{M} \subseteq \mathscr{A}, \rho$ the $H$-radical corresponding $\mathscr{K}$. The following are equivalent:

(1) $\rho$ has the ADS-property and $\mathscr{R}_{\rho}$ is hereditary;

(2) $\mathscr{M}$ satisfies condition $(\overline{\mathrm{G}})$ and $\mathscr{U} \mathscr{M}=\mathscr{U}(\overline{\mathscr{M}})_{k}$;

(3) $\mathscr{M}$ satisfies condition $(\overline{\mathrm{G}})$ and $\mathscr{U} \mathscr{M} \cap(\overline{\mathscr{M}})_{k}=0$.

4.17 Proposition. Let $\mathscr{A} \subseteq \mathscr{A}$ fulfil $(\overline{\mathrm{G}})$. Then the $H$-radical corresponding to $\mathscr{M}$ is r-hereditary if and only if $\overline{\mathscr{M}}$ is closed under essential extensions. 
Proof. The sufficiency follows from Proposition 4.14. Conversely, we show that the assumed two conditions imply $\left(2^{\prime}\right)$; the result then follows from Proposition 4.6. Consider thus $K \triangleleft I \triangleleft N \in \mathscr{A}$ with $0 \neq I / K \in \mathscr{M}$. By $(\overline{\mathrm{G}})$, there is an ideal $B \triangleleft N$ such that $B \subseteq K$ and $I / B \in \overline{\mathscr{M}}$. If $J / B$ is an ideal in $N / B$ which is maximal with respect to $J / B \cap I / B=0$, then $I / B \cong(I+J) / J \triangleleft \circ N / J$. Since $\overline{\mathscr{K}}$ is closed under essential extensions, $N / J \in \overline{\mathscr{M}}$. Furthermore, $N / J \neq 0$ and $I \cap J \subseteq B \subseteq K$.

4.18 Corollary. Let $M \subseteq \mathscr{A}, \rho$ the $H$-radical corresponding to $\mathscr{M}$. Then the following are equivalent:

(1) $\rho$ has the ADS-property and is r-hereditary;

(2) $\mathscr{M}$ satisfies $(\overline{\mathbf{G}})$ and $\overline{\mathscr{M}}=(\overline{\mathscr{K}})_{k}$;

(3) $\mathscr{M}$ satisfies $(\overline{\mathrm{G}})$ and $(N) \mathscr{M}=(N)(\overline{\mathscr{M}})_{k}$.

4.19 Proposition. Let $\mathscr{M} \subseteq \mathscr{A}$ such that $\overline{\mathscr{M}}$ is hereditary. Then the $H$ radical corresponding to $\mathscr{M}$ is r-hereditary if and only if $\overline{\mathscr{M}}$ is closed under essential extensions and $\mathscr{M}$ fulfils $(\overline{\mathbf{G}})$.

Proof. The necessity follows from Proposition 4.17. Conversely, if $\rho$ is $r$-hereditary, in view of Proposition 4.14, we only have to show that $\mathscr{M}$ fulfils $(\overline{\mathrm{G}})$. Let $K \triangleleft I \triangleleft N \in \mathscr{A}$ with $I / K \in \mathscr{M}$. Then $\rho I \subseteq K$. By Proposition 3.1, since $\mathscr{S}_{\rho}=\overline{\mathscr{M}}$ is hereditary and since $\rho$ is $r$-hereditary, $\rho I=I \cap \rho N \triangleleft N$. Hence $(\overline{\mathrm{G}})$ is fulfilled with $B=\rho I$.

In the above proposition, the hereditariness of $\overline{\mathscr{H}}$ cannot be weakened to regularity; see Example 6.15. Stronger results can be obtained, in a similar way, using $(\mathrm{G})$ instead of $(\overline{\mathrm{G}})$. Example 6.16 shows that if we assume only $(\bar{G})$ in the next proposition, then (iii) is weaker than the equivalent conditions (i) and (ii).

4.20 Proposition. Let $\mathscr{H} \subseteq \mathscr{A}$ fulfil $(\mathrm{G})$, $\rho$ the corresponding $H$-radical. The following are equivalent:

(i) $\rho$ is r-hereditary;

(ii) $\mathscr{\mathscr { K }}$ is closed under essential extensions;

(iii) $\mathscr{M}_{k} \subseteq \overline{\mathscr{M}}$.

Proof. (i) $\Leftrightarrow$ (ii) $\Rightarrow$ (iii) follows from Propositions 4.17 and 4.14. We only have to show that (iii) implies (ii). Let $I \triangleleft \circ N \in \mathscr{A}$ with $I \in \overline{\mathscr{M}}$. Then $I \cong \sum_{\text {subd }}\left(I / K_{\alpha} \mid K_{\alpha} \triangleleft I\right.$ with $I / K_{\alpha} \in \mathscr{M}$ and $\left.\bigcap_{\alpha} K_{\alpha}=0\right)$. By (G), for each $\alpha$ there is an ideal $B_{\alpha} \triangleleft N$ with $B_{\alpha} \subseteq K_{\alpha}$ and $I / B_{\alpha} \in \mathscr{M}$. For each $\alpha$, choose $C_{\alpha} / B_{\alpha} \triangleleft N / B_{\alpha}$ maximal with respect to $I / B_{\alpha} \cap C_{\alpha} / B_{\alpha}=0$. Then 
$I / B_{\alpha} \cong\left(I+C_{\alpha}\right) / C_{\alpha} \triangleleft \circ N / C_{\alpha} ;$ hence $N / C_{\alpha} \in \mathscr{M}_{k} \subseteq \overline{\mathscr{M}}$. Now $I \cap\left(\bigcap_{\alpha} C_{\alpha}\right) \subseteq$ $\bigcap_{\alpha} B_{\alpha} \subseteq \bigcap_{\alpha} K_{\alpha}=0$ and since $I$ is an essential ideal, $\bigcap_{\alpha} C_{\alpha}=0$. Thus $N \in \overline{\overline{\mathscr{H}}}=\overline{\mathscr{M}}$.

As in Propositions 4.17 and 4.19, the next two results can be verified.

4.21 Proposition. Suppose $\mathscr{M} \subseteq \mathscr{A}$ fulfils (G). Then $\mathscr{M}$ is closed under essential extensions if and only if $\mathscr{M}$ satisfies conditions $\left(1^{\prime}\right)$.

4.22 Proposition. Let $\mathscr{M} \subseteq \mathscr{A}$ be hereditary. Then $\mathscr{M}$ fulfils $\left(1^{\prime}\right)$ if and only if it is closed under essential extensions and satisfies $(\mathrm{G})$.

Comparing the above results, we are getting new information about the relationship between conditions $\left(1^{\prime}\right)$ and $\left(2^{\prime}\right)$. Under the presence of condition (G) on $\mathscr{M}$,

(1') is equivalent to $\mathscr{M}_{k} \subseteq \mathscr{M}$ (Proposition 4.21) and

$\left(2^{\prime}\right)$ is equivalent to $\mathscr{M}_{k} \subseteq \overline{\mathscr{M}}$ (Propositions 4.20 and 4.6).

In view of these equivalences, it is easy to see that $\left(1^{\prime}\right)$ is stronger than $\left(2^{\prime}\right)$ by considering Example 6.14.

Lastly we may mention that any subclass of $\mathscr{A}$ is always contained in a smallest KA-radical class which can be obtained by using, for example, the Tangeman-Kreiling construction [21]. It is well-known that for rings, if the starting class is hereditary, so is this radical class. Since the proof uses only the homomorphism theorems, it remains true for $\Omega$-groups. For completeness we only state:

4.23 Proposition. Let $\mathscr{D}$ be a hereditary homomorphically closed subclass of $\mathscr{A}$.

Let $\mathscr{D}_{1}:=\mathscr{D}$.

Let

$\mathscr{D}_{\lambda}:=\left\{N \in \mathscr{A} \mid\right.$ there exists an ideal $I \triangleleft N$ such that $I \in \mathscr{D}_{\lambda-1}$ and

$$
\left.N / I \in \mathscr{D}_{\lambda-1}\right\}
$$

for non-limit ordinals $\lambda$ and

$\mathscr{D}_{\lambda}:=\left\{N \in \mathscr{A} \mid N=\bigcup_{\alpha} I_{\alpha}\right.$ where $\left\{I_{\alpha}\right\}$ is an ascending chain of ideals $I_{\alpha}$ on $N$, each belonging to some $\left.\mathscr{D}_{\mu}, \mu<\lambda\right\}$

if $\lambda$ is a limit ordinal. 
Then $\mathscr{R}:=\bigcup_{\lambda \in \text { Ord }} \mathscr{D}_{\lambda}$ is a hereditary KA-radical class, in fact, the smallest $K A$-radical class which contains $\mathscr{D}$.

\section{Ideal hereditary radicals}

Following Rjabuhin [18], a radical $\rho$ on $\mathscr{A}$ is called ideal-hereditary if it is both $r$-and $s$-hereditary, that is $\rho I=\rho N \cap I$ for all $I \triangleleft N \in \mathscr{A}$.

5.1 Proposition. Let $\mathscr{M} \subseteq \mathscr{A}, \rho$ the corresponding $H$-radical. Then the following are equivalent:

(1) $\rho$ is ideal-hereditary;

(2) $\mathscr{K}$ satisfies condition (3) of Proposition 3.1 and any one of the conditions $\left(2^{\prime}\right),\left(4^{\prime}\right)$ or $\left(6^{\prime}\right)$ of Proposition 4.6;

(3) $\mathscr{M}$ satisfies any one of the conditions $\left(1^{\prime \prime}\right)$ to $\left(6^{\prime \prime}\right)$ of Proposition 4.4 and $\mathscr{S}_{\rho}$ is hereditary;

(4) $\rho$ is idempotent and both $\mathscr{R}_{\rho}$ and $\mathscr{S}_{\rho}$ are hereditary;

(5) $\mathscr{M}$ satisfies conditions (T), (3) of Proposition 3.1 and any one of conditions (1) to (6) of Remark 4.3;

(6) $\rho$ is a KA-radical with both $\mathscr{R}_{\rho}$ and $\mathscr{S}_{\rho}$ hereditary;

(7) $\rho$ is a KA-radical, $\mathscr{R}_{\rho}$ is hereditary and satisfies (*) $K \triangleleft I \triangleleft N \in \mathscr{A}$ and $K \in \mathscr{R}_{\rho}$ implies $\bar{K} \in \mathscr{R}_{\rho}$ where $\bar{K}$ is the ideal in $N$ generated by $K$;

(8) $\rho$ is a KA-radical with the ADS-property and $\mathscr{R}_{\rho}$ hereditary;

(9) $\mathscr{M}$ is regular, satisfies conditions $(\mathrm{T}),(\overline{\mathrm{G}})$ and any one of conditions (1) to (6) of Remark 4.3;

(10) $\mathscr{M}$ is regular, $\overline{\mathscr{M}}$ is closed under essential extensions and $\mathscr{M}$ fulfils $(\overline{\mathbf{G}})$.

Proof. (1) $\Leftrightarrow$ (2) by Propositions 3.1 and 4.6.

(3) $\Leftrightarrow(4)$ by Proposition 4.4.

(1) $\Rightarrow$ (4) by Propositions 3.1 and 4.1 .

(4) $\Rightarrow(5)$ by Propositions 2.4, 3.1 and Remark 4.3.

(5) $\Rightarrow(6)$ since $\mathscr{S}_{p}$ hereditary implies $\rho$ complete.

(6) $\Rightarrow$ (7) only (*) needs verification. If $K \triangleleft I \triangleleft N \in \mathscr{A}$ is as required in (*), then $K \subseteq \rho I \subseteq \rho N \cap I \triangleleft N$. Thus $\bar{K} \subseteq \rho N \cap I \subseteq \rho N$ and the hereditariness of $\mathscr{R}_{\rho}$ yields $\bar{K} \in \mathscr{R}_{\rho}$.

$(7) \Rightarrow(8)$ for $I \triangleleft N \in \mathscr{A}$, we have $\rho I \triangleleft I \triangleleft N$ and $\rho I \in \mathscr{R}_{\rho}$. By (*) $\overline{\rho I} \in \mathscr{R}_{\rho}$; hence $\overline{\rho I} \subseteq \rho I$.

$(8) \Leftrightarrow(9)$ by Propositions 2.2, 2.4, 3.2 and Remark 4.3.

$(8) \Leftrightarrow(1)$, well-known. 
$(10) \Leftrightarrow(8)$ by Corollary 4.16 and Proposition 2.2 .

Condition $(*)$ holds obviously if the $\triangleleft$-relation is transitive on $\mathscr{A}$. The following proposition shows that it holds in other important cases.

5.2 Proposition. Let $\mathscr{A}$ be a universal class of alternative rings or groups. If $\mathscr{M} \subseteq \mathscr{A}$ is regular, then $\mathscr{R}=\mathscr{U} \mathscr{M}$ satisfies (*). Thus every complete $H$ radical on $\mathscr{A}$ fulfils $(*)$.

Proof. The proof depends on the following result proved by Krempa [8] for alternative rings and is easy to verify for groups:

Let $K \triangleleft \bar{K} \triangleleft N$ with $\bar{K}$ the ideal of $N$ generated by $K$. Then every non-zero homomorphic image of $\bar{K}$ contains a non-zero ideal which is a homomorphic image of $K$.

We proceed with the proof of the proposition. Let $K \triangleleft I \triangleleft N$ with $K \in \mathscr{R}$. If $\bar{K} \notin \mathscr{R}$, then $\bar{K}$ has a non-zero homomorphic image, say $\bar{K} / B$, such that $0 \neq \bar{K} / B \in \mathscr{M}$. By the above-mentioned result, $\bar{K} / B$ contains a non-zero ideal, say $C / B$, which is a homomorphic image of $K$. Since $\mathscr{M}$ is regular, $C / B$ and hence $K$, has a non-zero homomorphic image in $\mathscr{M}$ which contradicts $K \in \mathscr{R}=\mathscr{U} \mathscr{H}$.

5.3 COROLLARY. If $\mathscr{A}$ is a universal class of alternative rings or groups, (7) in Proposition 5.1 can be weakened to: $\rho$ is a KA-radical and $\mathscr{R}_{\rho}$ is hereditary.

5.4 Remark. (7) and (8) in Proposition 5.1 cannot be weakened (as (6) to (4)) by claiming only that $\rho$ is idempotent or complete (and not necessarily KA) -Example 6.1 provides us with an idempotent $H$-radical $\rho$ with ADS and $\mathscr{R}_{\rho}$ hereditary and satisfying $(*)$ (since, in 6.1 , normality is transitive) but $\rho$ is not complete. Also, Example 6.2 shows that ADS, regularity and (*) do not imply $\mathscr{S}_{\rho}$ hereditary. Furthermore, Example 6.6 shows that the hereditariness of both $\mathscr{R}_{\rho}$ and $\mathscr{S}_{\rho}$ do not imply, even in the presence of ADS and (*), that $\rho$ is idempotent. These observations confirm the fact already mentioned in Section 3 that the ADS is not a very useful property for $\mathrm{H}$ radicals which are not $\mathrm{KA}$.

Our next result generalizes Theorem 1 of Anderson, Kaarli and Weigandt [1] in that we use condition $(G)$ where they have used condition $(F)$.

5.5 Proposition. If $\mathscr{M} \subseteq \mathscr{A}$ is regular, satisfies (G) and $\mathscr{M}_{k} \subseteq \overline{\mathscr{K}}$, then the corresponding $H$-radical is ideal-hereditary (and thus a $K A$-radical).

Proof. By Propositions 4.20 and 5.1(10). 
The converse is not true since $(G)$ is not subdirectly invariant (compare Example 6.7).

5.6 Corollary. $\mathscr{M} \subseteq \mathscr{A}$ is the semisimple class of an ideal-hereditary $K A$-radical if and only if $\mathscr{M}$ is regular, closed under subdirect sums and essential extensions and satisfies condition $(\mathrm{G})$.

For a KA-radical $\rho$, the next implications illustrate some of the characterizing conditions of properties which we have considered. As usual, if $K \triangleleft I \triangleleft N \in \mathscr{A}$, then $\bar{K}$ means the ideal of $N$ generated by $K$. In this diagram, each statement to the right of an equivalence sign $(\Leftrightarrow)$, should be followed by "... for all $I \triangleleft N \in \mathscr{A} "$ :

$$
\begin{aligned}
& \rho \text { ideal-hereditary } \quad \Leftrightarrow \rho I=\rho N \cap I \quad \Leftrightarrow \rho I=\rho N \cap I \\
& \Downarrow \\
& \rho \text { has the ADS-property } \Leftrightarrow \rho(\overline{\rho(I)})=\rho N \cap \overline{\rho(I)} \Leftrightarrow \rho I=\rho N \cap \overline{\rho(I)} \\
& \Downarrow \\
& \rho s \text {-hereditary } \\
& \Leftrightarrow \rho(\rho I)=\rho N \cap \rho I \quad \Leftrightarrow \rho I=\rho N \cap \rho I .
\end{aligned}
$$

In 4.1 , the condition $\rho(\rho N \cap I) \subseteq \rho I$ has appeared. If $\rho$ is a KA-radical, the following can easily be verified:

$$
\rho(\rho N \cap I)=\rho N \cap I \text { for all } I \triangleleft N \in \mathscr{A} \Leftrightarrow \mathscr{R}_{\rho} \text { is hereditary and }
$$

and

$$
\begin{aligned}
& \rho(\rho N \cap I)=\rho I \text { for all } I \triangleleft N \in \mathscr{A} \Leftrightarrow \rho(\rho N \cap I) \supseteq \rho I \text { for all } I \triangleleft N \\
& \Leftrightarrow \mathscr{S}_{\rho} \text { is hereditary. }
\end{aligned}
$$

\section{Examples}

In order to avoid a lot of unnecessary repetitions, we first give a summary of the main implications between the conditions which we considered. In the examples we will then only mention the strongest one (unless we want to emphasize something specific). In our scheme, $i$ represents any element from the set $\{1,2,3,4,5,6\}$ and $\rho$ is always the $H$-radical corresponding to $\mathscr{M}$ (as in the examples that follow).

6.1 Let $\mathscr{A}$ be the variety of all abelian groups. In this case, every subgroup is normal; thus every subclass of $\mathscr{A}$ satisfies (F). Let

$$
\mathscr{M}:=\{N \in \mathscr{A} \mid N=0 \text { or } N \text { is finite }\} \text {. }
$$


is closed both under essential extensions and subdirect products, hence it fulfils $\left(3^{\prime}\right)$ (since $(G)$ is valid). It can easily be verified that

$$
\rho N= \begin{cases}N & \text { if } N \text { is finite, } \\ 0 & \text { if } N \text { is infinite; }\end{cases}
$$

hence $\rho$ is the lower $H$-radical in $\mathscr{A}$ determined by the class of all finite abelian groups. Clearly $\rho$ is $r$-hereditary. Thus $\rho$ is idempotent but not complete since $\mathscr{S}_{\rho}=\mathscr{\mathscr { M }}=\mathscr{M}$ is not regular. In fact, $\mathscr{M}$ is not even an $r$-class.

6.2 Let again $\mathscr{A}$ be the variety of all abelian groups; as above every subclass of $\mathscr{A}$ satisfies (F). For a prime $p$, let $c(p)$ and $c\left(p^{\infty}\right)$ denote the cyclic group of order $p$ and the infinite quasicyclic group respectively (compare Example 1 in Divisky [4]). Let $\mathscr{M}$ be the subclass of $\mathscr{A}$ which consists of $0, c(p)$ and $c\left(p^{\infty}\right)$ where $p$ runs through all the primes $p$. $\mathscr{M}$ is regular since every proper ideal of $c\left(p^{\infty}\right)$ can be mapped homomorphically onto $c(p)$. Consequently $\rho$ is complete and has the ADS-property. Using Proposition 3.1, we now show that $\rho$ is not $s$-hereditary. For a fixed prime $p$, let $H_{2}:=\left\{0,1 / p^{2}, 2 / p^{2}, \ldots\left(p^{2}-1\right) / p^{2}\right\}$. Then $c\left(p^{2}\right) \cong H_{2} \triangleleft c\left(p^{\infty}\right) \in \mathscr{M}$, but $H_{2} \notin \overline{\mathscr{M}}$. Using the well-known result that every abelian group has a homomorphic image of the form $c(q)$ or $c\left(q^{\infty}\right)$ for a suitable prime $q$, we have $\mathscr{R}_{\rho}=\mathscr{U} \mathscr{M}=0$; thus $\mathscr{R}_{\rho}$ is hereditary. The only KA-radical corresponding to $\mathscr{R}_{\rho}=0$ being the trivial one, we can conclude that $\rho$ is not $\mathrm{KA}$; hence not idempotent and thus not $r$-hereditary. From Proposition 4.20 we obtain $\mathscr{M}_{k} \nsubseteq \overline{\mathscr{K}}$. Also notice that $\mathscr{M}$ is not closed under essential $\mathscr{M}$-representable extensions. Indeed, if $H_{1}:=\{0,1 / p, 2 / p, \ldots,(p-1) / p\}$ for a fixed prime $p$, then $c(p) \cong H_{1} \triangleleft H_{2}\left(H_{2}\right.$ is defined above) and $H_{1}$ is $\mathscr{M}$-representable and essential in $H_{2}$, but $H_{2} \notin \mathscr{M}$. Obviously $\rho N \subseteq \mathscr{R}_{\rho}(N)=0$ does not hold in general; moreover, $\rho$ is not hereditarily idempotent on $\mathscr{S}_{\rho}$ (since $H_{2} \triangleleft c\left(p^{\infty}\right) \in \mathscr{S}_{\rho}$ but $\left.\rho \rho H_{2} \neq \rho H_{2}\right)$.

6.3 (Leavitt [12]). Let $\mathscr{Z}_{2}$ be the two-element field and let $\mathscr{Z}_{2}^{0}$ be the zeroring on $\mathscr{Z}_{2}^{+}$. Let $K$ be the ring generated over $\mathscr{Z}_{2}$ by $\{e, x\}$ where $e^{2}=e$, $e x=x e=x$ and $x^{2}=0$. Let

$$
\mathscr{A}:=\left\{0, \mathscr{Z}_{2}, \mathscr{Z}_{2}^{0}, \mathscr{Z}_{2} \oplus \mathscr{Z}_{2}^{0}, \mathscr{Z}_{2}^{0} \oplus \mathscr{Z}_{2}^{0}, K, K \oplus \mathscr{Z}_{2}^{0}\right\} .
$$

$\mathscr{A}$ is a universal class of (associative and commutative) rings since the only non-trivial ideal of $K$ is the ideal in $K$ generated by $x$. But this ideal is isomorphic to $\mathscr{Z}_{2}^{0}$ and $K / \mathscr{Z}_{2}^{0} \cong \mathscr{Z}_{2}$. Let

$$
\mathscr{M}:=\left\{0, \mathscr{Z}_{2}, \mathscr{Z}_{2}^{0} \oplus \mathscr{Z}_{2}^{0}\right\} .
$$




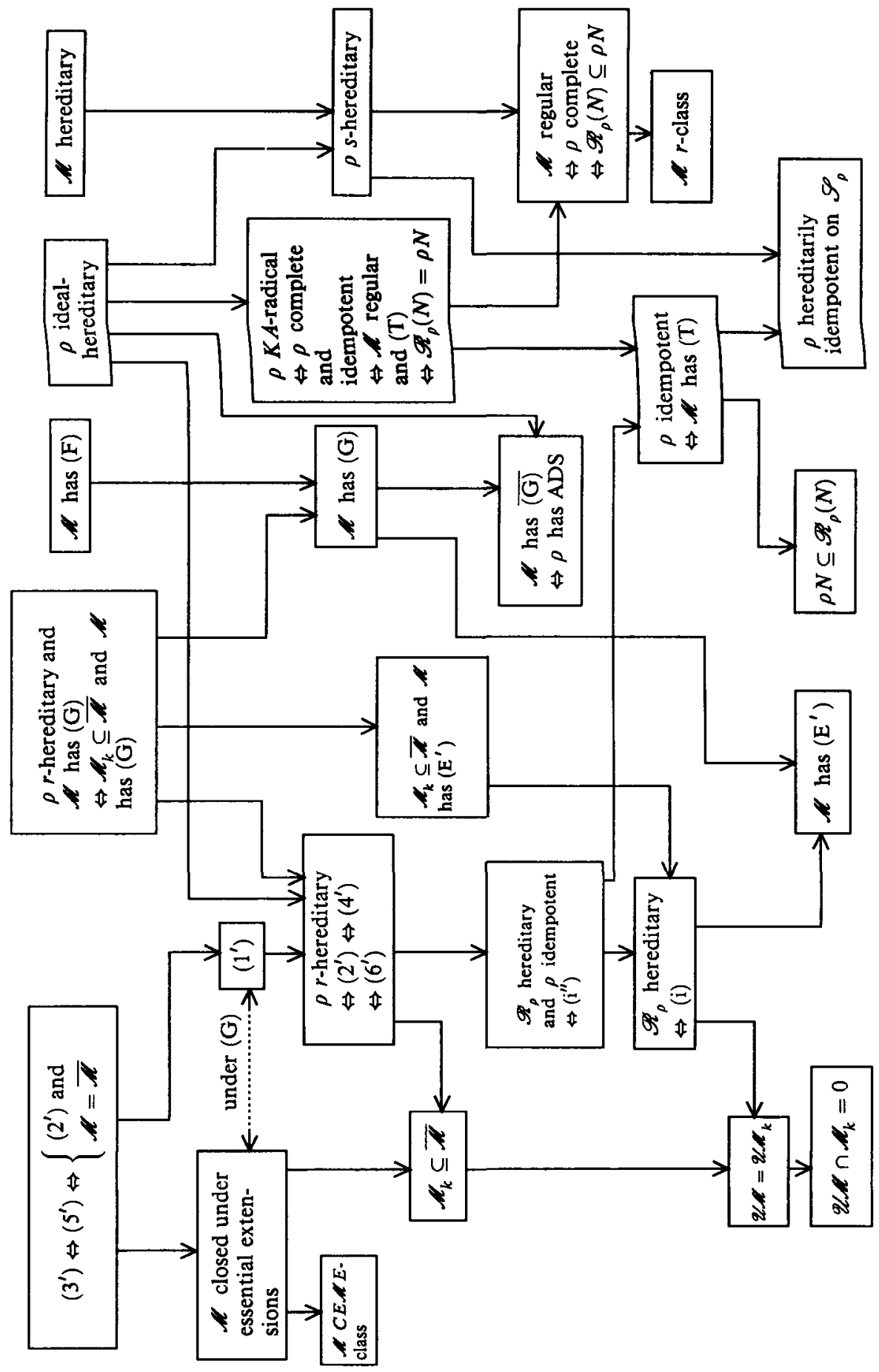


To simplify the verification of the conditions, we provide

\begin{tabular}{c|c|c|c|c|c|c|c}
$N$ & 0 & $\mathscr{Z}_{2}$ & $\mathscr{Z}_{2}^{0}$ & $\mathscr{Z}_{2} \oplus \mathscr{Z}_{2}^{0}$ & $\mathscr{Z}_{2}^{0} \oplus \mathscr{Z}_{2}^{0}$ & $K$ & $K \oplus \mathscr{Z}_{2}^{0}$ \\
\hline$\rho N$ & 0 & 0 & $\mathscr{Z}_{2}^{0}$ & $\mathscr{Z}_{2}^{0}$ & 0 & $\mathscr{Z}_{2}^{0}$ & $\mathscr{Z}_{2}^{0} \oplus \mathscr{Z}_{2}^{0}$ \\
$\mathscr{R}_{\rho}(N)$ & 0 & 0 & $\mathscr{Z}_{2}^{0}$ & $\mathscr{Z}_{2}^{0}$ & $\mathscr{Z}_{2}^{0} \oplus \mathscr{Z}_{2}^{0}$ & $\mathscr{Z}_{2}^{0}$ & $\mathscr{Z}_{2}^{0} \oplus \mathscr{Z}_{2}^{0}$
\end{tabular}

The relation of being an ideal in $\mathscr{A}$ is transitive, hence $\mathscr{M}$ satisfies (F). Furthermore, $\mathscr{M}=\overline{\mathscr{M}}, \mathscr{R}_{\rho}=\left\{0, \mathscr{Z}_{2}^{0}\right\}$ is hereditary and $\rho N \subseteq \mathscr{R}_{\rho}(N)$ for all $N \in \mathscr{A}$; hence $\rho$ satisfies condition $\left(4^{*}\right)$ of Remark 2.3. However, $\mathscr{M}$ is not an $r$-class, not a CE $\mathscr{K}$ E-class, $\mathscr{M}_{k} \nsubseteq \mathscr{M}$ and $\rho$ is not idempotent (but hereditarily idempotent on $\left.\mathscr{S}_{\rho}=\mathscr{M}\right)$. Lastly note that $0 \neq \mathscr{R}_{\rho}\left(\mathscr{Z}_{2}^{0} \oplus \mathscr{Z}_{2}^{0}\right) \in \mathscr{M}$ so $\rho$ is not complete.

6.4 (Leavitt [12]). Let $\mathscr{A}$ be the same universal class as in Example 6.3. In this case, let $\mathscr{M}=\left\{0, \mathscr{Z}_{2}, \mathscr{Z}_{2}^{0} \oplus \mathscr{Z}_{2}^{0}, \mathscr{Z}_{2} \oplus \mathscr{Z}_{2}^{0}\right\}$. We then have

\begin{tabular}{c|c|c|c|c|c|c|c}
$N$ & 0 & $\mathscr{Z}_{2}$ & $\mathscr{Z}_{2}^{0}$ & $\mathscr{Z}_{2} \oplus \mathscr{Z}_{2}^{0}$ & $\mathscr{Z}_{2}^{0} \oplus \mathscr{Z}_{2}^{0}$ & $K$ & $K \oplus \mathscr{Z}_{2}^{0}$ \\
\hline$\rho N$ & 0 & 0 & $\mathscr{Z}_{2}^{0}$ & 0 & 0 & $\mathscr{Z}_{2}^{0}$ & $\mathscr{Z}_{2}^{0}$ \\
$\mathscr{R}_{\rho}(N)$ & 0 & 0 & $\mathscr{Z}_{2}^{0}$ & $\mathscr{Z}_{2}^{0}$ & $\mathscr{Z}_{2}^{0} \oplus \mathscr{Z}_{2}^{0}$ & $\mathscr{Z}_{2}^{0}$ & $\mathscr{Z}_{2}^{0} \oplus \mathscr{Z}_{2}^{0}$
\end{tabular}

Then $\mathscr{K}$ has (F) (as above), $\mathscr{M}=\overline{\mathscr{K}}, \mathscr{R}_{\rho}=\left\{0, \mathscr{Z}_{2}^{0}\right\}$ is hereditary and $\rho$ is idempotent. $\mathscr{M}$ is not an $r$-class, not a CE $\mathscr{K}$ E-class, $\mathscr{M}_{k} \nsubseteq \mathscr{M}$ and $\rho$ is not $r$ hereditary (since $\rho\left(K \oplus \mathscr{Z}_{2}^{0}\right) \cap\left(\mathscr{Z}_{1}^{0} \oplus \mathscr{Z}_{2}^{0}\right) \cong \mathscr{Z}_{2}^{0} \cap \mathscr{Z}_{2}^{0} \oplus \mathscr{Z}_{2}^{0} \cong \mathscr{Z}_{2}^{0} \nsubseteq 0=\rho\left(\mathscr{Z}_{2}^{0} \oplus\right.$ $\left.\mathscr{Z}_{2}^{0}\right)$ ). Thus conditions (a) and (b) hold but not (c) (compare Proposition 4.1). Once again we have $0 \neq \mathscr{R}_{\rho}(N) \in \mathscr{M}$ for $N \in\left\{\mathscr{Z}_{2}^{0} \oplus \mathscr{Z}_{2}^{0}, K \oplus \mathscr{Z}_{2}^{0}\right\}$.

6.5 Using Lemma 1 and Lemma 2 in Rjabuhin [20], construct rings $A_{1}$, $A_{2}, A_{3}, A_{4}$ such that $A_{i}$ is the only proper ideal of $A_{i+1}$ for $i=1,2,3$ and $A_{1}$ and $B_{i+1}=A_{i+1} / A_{i}$ are non-isomorphic simple rings. Let $\mathscr{A}:=$ $\left\{0, A_{1}, A_{2}, A_{3}, A_{4}, B_{2}, B_{3}, B_{4}\right\}$. Then the lower KA-radical $\mathscr{R}$ in $\mathscr{A}$ generated by $A_{2}$ is $\mathscr{R}=\left\{0, A_{2}, B_{2}\right\}$ with $\mathscr{S} \mathscr{R}=\left\{0, A_{1}, A_{4}, B_{3}, B_{4}\right\}$. Both $\mathscr{R}$ and $\mathscr{S} \mathscr{R}$ are non-hereditary, but $\mathscr{R}$ satisfies condition (c) of Proposition 4.1.

6.6 Let $\mathscr{A}$ be the variety of associative rings. Leavitt [9] has given an example of a class $\mathscr{H}$ of simple prime rings, not all with an identity, such that $\mathscr{R}_{\rho}=\mathscr{U} \mathscr{M}$ is hereditary. Clearly $\mathscr{M}$ is hereditary and fulfils (F) since it consists of prime rings. Andrunakievič [2] has shown that a class $\mathscr{K}$ of simple rings consists of rings with identity if and only if $\mathscr{U} \mathscr{K}$ is hereditary and $\overline{\mathscr{K}}=\mathscr{S} \mathscr{U K}$. Consequently, for our choice of $\mathscr{M}$, we have $\overline{\mathscr{K}} \subseteq \mathscr{S} \mathscr{U} \mathscr{K}$, 
implying that $\rho$ is not a KA-radical (compare Corollary 2.5) and thus not idempotent and not $r$-hereditary. By Proposition 4.20 we obtain $\mathscr{M}_{k} \nsubseteq \overline{\mathscr{M}}$. Trivially, $\mathscr{K}$ is not a CE $\mathscr{K}$ E-class.

6.7 Let $\mathscr{A}$ be the variety of all (right) near-rings and let $\mathscr{M}$ be the class of all subdirectly irreducible near-rings. Since every non-zero near-ring has a non-zero homomorphic image in $\mathscr{M}, \mathscr{M}$ is regular and by the well-known Birkhoff theorem, $\mathscr{M}=\mathscr{A}$. Thus $\rho$ is the trivial radical, hence an idealhereditary KA-radical. $\mathscr{M}$ fulfils $(\overline{\mathrm{G}})$ but is neither hereditary nor subdirectly closed nor does it fulfil (G): condition (G) for $\mathscr{M}$ would imply by Theorem 3.2 in [22] that $\mathscr{K}$ consists of semiprime near-rings which is certainly not the case. We show now that $\mathscr{M}$ fulfils both (1') and $\mathscr{M}_{k} \subseteq \mathscr{M}$ :

To show (1') consider $K \triangleleft I \triangleleft N \in \mathscr{A}$ with $0 \neq I / K \in \mathscr{M}$. Choose $J \triangleleft N$ maximal with respect to $I \cap J \subseteq K$. Clearly $N / J \neq 0$; we show $N / J$ is subdirectly irreducible. Let $\left\{B_{\alpha} / J\right\}_{\alpha}$ be the class of all non-zero ideals of $N / J$. By our choice of $J, I \cap B_{\alpha} \nsubseteq K$ for each $\alpha$. Hence, for each $\alpha$, $0 \neq\left(\left(I \cap B_{\alpha}\right)+K\right) / K \triangleleft I / K$ and since $I / K$ is subdirectly irreducible, there is an $x+K \in I / K$ with $x \notin K$ and $x \in I \cap B_{\alpha}$ for all $\alpha$. Thus $0 \neq x+J \in B_{\alpha} / J$ for each $\alpha$.

To show $\mathscr{M}_{k} \subseteq \mathscr{M}$ consider $I \triangleleft \circ N$ with $I$ subdirectly irreducible. If $N$ is not subdirectly irreducible, there are ideals $B_{\alpha} \triangleleft N$ such that $\bigcap_{\alpha} B_{\alpha}=0$. Without loss of generality, assume $B_{\alpha} \neq 0$ for each $\alpha$. Since $I$ is an essential ideal in $N, 0 \neq I \cap B_{\alpha} \triangleleft I$ for each $\alpha$, but $\bigcap_{\alpha}\left(I \cap B_{\alpha}\right)=0$ which contradicts our choice of $I$.

6.8 Leavitt [12]. Let $H$ be the ring generated over $\mathscr{Z}_{2}$ by the commutative symbols $\{x, y, z, u\}$ with relations $x^{2}=x, x y=x, y^{2}=u z=0, x z=y$, $y z=x, z^{2}=y, u^{2}=u, u x=x$ and $u y=y$. As usual, by $(r)$ we denote the ideal in $H$ generated by $r \in H$. The only non-trivial ideals in $H$ are $A=(z), B=(u)$ and $C=(x)=(y)$ and the only non-trivial ideal in $(x)$ is $D=\{0, x\}$. The non-trivial homomorphic images of accessible subrings of $H$ are $H / A \cong B / C \cong \mathscr{Z}_{2} \cong D, H / B \cong A / C \cong C / D \cong \mathscr{Z}_{2}^{0}$ and $H / C \cong \mathscr{Z}_{2} \oplus \mathscr{Z}_{2}^{0}$.

Let $K$ be the ring as defined in Example 6.3. Recall that $K$ has only one non-trivial ideal which is isomorphic to $\mathscr{Z}_{2}^{0}$ and $K / \mathscr{Z}_{2}^{0} \cong \mathscr{Z}_{2}$.

Let $\mathscr{A}$ be the (universal) class of all homomorphic images of accessible subrings of $H$ and $K$, that is $\mathscr{A}=\left\{H, 0, A, B, C, \mathscr{Z}_{2}, \mathscr{Z}_{2}^{0}, \mathscr{Z}_{2} \oplus \mathscr{Z}_{2}^{0}, K\right\}$. In this case, let $\mathscr{M}:=\left\{0, B, A, \mathscr{Z}_{2}^{0}\right\}$. Then

\begin{tabular}{c|c|c|c|c|c|c|c|c|c}
$N$ & $H$ & 0 & $A$ & $B$ & $C$ & $\mathscr{Z}_{2}$ & $\mathscr{Z}_{2} \oplus \mathscr{Z}_{2}^{0}$ & $\mathscr{Z}_{2}^{0}$ & $K$ \\
\hline$\rho N$ & $B$ & 0 & 0 & 0 & $\mathscr{Z}_{2}$ & $\mathscr{Z}_{2}$ & $\mathscr{Z}_{2}$ & 0 & $K$ \\
$\mathscr{R}_{\rho}(N)$ & 0 & 0 & 0 & 0 & $\mathscr{Z}_{2}$ & $\mathscr{Z}_{2}$ & $\mathscr{Z}_{2}$ & 0 & $K$
\end{tabular}


$\mathscr{M}=\overline{\mathscr{M}}$ is regular, fulfils $\left(\mathrm{E}^{\prime}\right)$ and $\mathscr{U} \mathscr{M} \cap \mathscr{M}_{k} \neq 0 . \rho$ is now idempotent (but hereditarily idempotent on $\overline{\mathscr{K}})$, not $s$-hereditary and does not fulfil condition (c) of Proposition $4.1(\rho(\rho H \cap A) \nsubseteq \rho(A))$. Note also that $\mathscr{R}_{\rho}=\left\{0, \mathscr{Z}_{2}, K\right\}$ is not hereditary, $\mathscr{M}$ is not a CE $\mathscr{M}$ E-class, does not fulfil $(\overline{\mathrm{G}})$ and $\rho N \nsubseteq \mathscr{R}_{\rho}(N)$.

6.9 Leavitt [12]. Restricting Example 6.8 to the universal class $\mathscr{A}^{\prime}:=$ $\mathscr{A}-\{K\}$, most of the properties carry over. The only differences are: $\mathscr{R}_{\rho}:=$ $\left\{0, \mathscr{Z}_{2}\right\}$ is not hereditary, implying $\mathscr{U} \mathscr{M}=\mathscr{U} \mathscr{M}_{k}$; as before $\mathscr{M}_{k} \nsubseteq \mathscr{M}=\overline{\mathscr{M}}$.

6.10 Leavitt [12]. Let $X$ be the ring generated over $\mathscr{Z}_{3}$ by $\{f, y\}$ where $f^{2}=f, f y=y f=y, y^{2}=0$. The only non-trivial ideal of $X$ is $(y)=$ $\{0, y, y+y\} \cong \mathscr{Z}_{3}^{0}$, and $X /(y) \cong \mathscr{Z}_{3}$. Let $\mathscr{A}$ be the (universal) class consisting of all homomorphic images of ideals of $X$ and $K$ (where $K$ is the ring defined in Example 6.3), that is $\mathscr{A}=\left\{K, 0, \mathscr{Z}_{2}^{0}, \mathscr{Z}_{2}, X, \mathscr{Z}_{3}^{0}, \mathscr{Z}_{3}\right\}$ Let $\mathscr{M}:=\left\{0, \mathscr{Z}_{2}^{0}, \mathscr{Z}_{3}^{0}, \mathscr{Z}_{3}\right\}$ Then

\begin{tabular}{c|c|c|c|c|c|c|c}
$N$ & 0 & $K$ & $\mathscr{Z}_{2}^{0}$ & $\mathscr{Z}_{2}$ & $X$ & $\mathscr{Z}_{3}^{0}$ & $\mathscr{Z}_{3}$ \\
\hline$\rho N$ & 0 & $K$ & 0 & $\mathscr{Z}_{2}$ & $\mathscr{Z}_{3}^{0}$ & 0 & 0 \\
$\mathscr{R}_{\rho}(N)$ & 0 & $K$ & 0 & $\mathscr{Z}_{2}$ & 0 & 0 & 0
\end{tabular}

Since the relation of being an ideal is transitive in $\mathscr{A}, \mathscr{M}$ satisfies $(\mathrm{F}), \mathscr{M}$ is hereditary, not a CE $\mathscr{M}$ E-class, $\mathscr{U} \mathscr{M} \cap \mathscr{M}_{k} \neq 0$ and $\rho$ satisfies condition (c) of Proposition 4.1. However, $\rho$ is not idempotent (in fact, $\rho X \nsubseteq \mathscr{R}_{\rho}(X)$ ) and $\mathscr{R}_{\rho}=\left\{0, K, \mathscr{Z}_{2}\right\}$ is not hereditary.

6.11 Leavitt [12]. Let $\mathscr{A}$ be the universal class of all rings and let $\rho$ be the KA-radical with $\mathscr{R}_{\rho}$ the class of all idempotent rings. As is well-known, both $\mathscr{R}_{\rho}$ and $\mathscr{S}_{\rho}$ are non-hereditary. We show that $\rho$ does not satisfy condition (c) of Proposition 4.1. Indeed, consider the ring $H$ from Example 6.8. Since $B^{2}=B$ and $D^{2}=D$, we have $\rho(\rho H \cap A)=\rho(B \cap A)=\rho C=D$ but $\rho A=0$.

6.12 Let $\mathscr{A}$ be the class of all associative rings and let $\mathscr{M}$ be a special class (that is a hereditary class of prime rings which is closed under essential extensions). Since $\mathscr{M}$ consists of prime rings, it satisfies (F) and by Proposition 4.22 also $\left(1^{\prime}\right)$. Consequently $\rho$ is an ideal-hereditary KA-radical. Clearly $\mathscr{M}$ is not subdirectly closed.

6.13 Let $\mathscr{A}$ be the class of all associative rings, Leavitt [11], Theorem 4, has constructed a regular class $\mathscr{M}$ in $\mathscr{A}$ for which $\mathscr{M}=\mathscr{M}_{k}$ but $\mathscr{R}_{\rho}=\mathscr{U} \mathscr{M}$ is not hereditary. $\mathscr{K}$ does not satisfy $\left(E^{\prime}\right)$ (compare Proposition 4.11). Using Proposition 7 in [11] and the definition of the class $\mathscr{M}$, it follows that $\mathscr{M}$ is not hereditary. Indeed, there is a ring $A$ such that $\mathscr{Z}_{p}^{0} \triangleleft \circ A$ from which $A \in \mathscr{M}$ follows. But $J$, which is a direct sum of $n$ copies of $\mathscr{Z}_{p}^{0}$ and is and 
ideal in $A$, is not in $\mathscr{M}$. However, following Proposition 3.1(3), we can show that $\rho$ is $s$-hereditary: take $I \triangleleft N \in \mathscr{M}$. Then there is some index $i$ with $N$ accessible in $L \in \mathscr{K}_{i}^{c}$, hence $I$ is accessible in $L$. Then either $I \in \mathscr{M}$ or $I$ has $\mathscr{Z}_{p}^{0}$ as a proper direct summand. If $I$ is a direct sum of $\mathscr{Z}_{p}^{0}$ 's, then $I \in \overline{\mathscr{M}}$. Otherwise, we may represent $I$ as a direct sum of $J_{\alpha}$ 's with exactly one $J_{\alpha}$ not containing $\mathscr{Z}_{p}^{0}$ as a direct summand and all other $J_{\alpha}$ 's being isomorphic to $\mathscr{Z}_{p}^{0}$. Thus all $J_{\alpha}$ 's are in $\mathscr{M}$ from which $I \in \overline{\mathscr{M}}$ follows.

6.14 Let $\mathscr{A}$ be the class of all finite abelian groups. Then trivially every subclass fulfils (F). Let $\mathscr{M}:=\left\{c\left(2^{n}\right) \mid n=1,2,3, \ldots\right\} \cup\{c(2) \times c(2)\} \cup\{0\}$. Then $\overline{\mathscr{M}}$ consists exactly of all abelian groups of order $2^{n}, n=1,2,3, \ldots$. For any $N \in \mathscr{A}$, we have two possibilities. If $|N| \neq 2^{n}$ for all $n$, then $N$ is the direct sum of cyclic groups, not all with order of the form $2^{k}$ and the elements of $\mathscr{M}$ cannot be embedded in $N$ as essential ideals. If $|N|=2^{n}$ for some $n$, then $N \in \overline{\mathscr{K}}$. It thus follows that $\mathscr{M}_{k} \subseteq \overline{\mathscr{M}}$; consequently $\mathscr{M}$ satisfies $\left(2^{\prime}\right)$. But, for example, $c(2) \times c(2)$ is essential and $\mathscr{K}$-representable in $c(4) \times c(4) \notin \mathscr{M}$. Hence $\mathscr{M}$ is not a CE $\mathscr{H}$ E-class (and thus also $\mathscr{M}_{k} \nsubseteq \mathscr{M}$ ). $\overline{\mathscr{H}}=\mathscr{S}_{\rho}$ is hereditary; hence $\rho$ is an ideal-hereditary KA-radical.

6.15 Let $\mathscr{A}$ be the variety of all $o$-symmetric (left) near-rings. Let $\mathscr{N}$ be the nil radical class. As is well-known, $\mathscr{N}$ is a hereditary (= $r$-hereditary) KA-radical class, but Kaarli [7] has shown that $\mathscr{S} \mathscr{N}$ is not hereditary. Hence $N$ does not satisfy the ADS-property. By Corollary $4.8, \mathscr{S N}$ satisfies $\left(i^{\prime}\right)$ for $i=1,2, \ldots, 6$. Furthermore, by Proposition 4.11, $\mathscr{S N}$ fulfils $\left(\mathrm{E}^{\prime}\right)$; hence $\left(E^{\prime}\right)$ does not imply $(\overline{\mathbf{G}})$, even for subdirectly closed classes. Moreover, by Proposition 4.14, $\mathscr{S N}$ is closed under essential extensions.

6.16 By Leavitt [11], Corollary 2, there is a ring $B$ with a single proper ideal $D=\mathscr{Z}_{p}^{0} \oplus \mathscr{Z}_{p}^{0} \oplus \mathscr{Z}_{p}^{0}$ such that $B / D \cong F$ where $F$ is a field of degree 3 over $\mathscr{Z}_{p}$. Let $\mathscr{A}:=\left\{0, F, \mathscr{Z}_{p}^{0}, \mathscr{Z}_{p}^{0} \oplus \mathscr{Z}_{p}^{0}, D, B\right\}$ and $\mathscr{M}:=\left\{0, \mathscr{Z}_{p}^{0} \oplus \mathscr{Z}_{p}^{0}\right\}$. Then obviously $(\overline{\mathrm{G}})$ is fulfilled, implying $\left(\mathrm{E}^{\prime}\right)$ for $\overline{\mathscr{M}}$. But there is no ideal of $B$ sending $D$ into $\mathscr{M}$, hence $\left(\mathrm{E}^{\prime}\right)$ does not hold for $\mathscr{M}$. From the following diagram, it can be verified that $\mathscr{K}$ is not an r-class, $\rho$ is idempotent and $\mathscr{M}_{k}=\mathscr{M} \subseteq \overline{\mathscr{M}}$; but $\mathscr{M}$ is not closed under essential extensions (consider $D$ and $B: D \in \overline{\mathscr{M}}$ is essential in $B$ ).

\begin{tabular}{c|l|l|l|c|c|c}
$N$ & 0 & $F$ & $\mathscr{Z}_{p}^{0}$ & $\mathscr{Z}_{p}^{0} \oplus \mathscr{Z}_{p}^{0}$ & $D$ & $B$ \\
\hline$\rho N$ & 0 & $F$ & $\mathscr{Z}_{p}^{0}$ & 0 & 0 & $B$ \\
$\mathscr{R}_{\rho}(N)$ & 0 & $F$ & $\mathscr{Z}_{p}^{0}$ & $\mathscr{Z}_{p}^{0} \oplus \mathscr{Z}_{p}^{0}$ & $D$ & $B$
\end{tabular}

In closing, the following remark seems to be in order. The examples 6.3 to 6.5, 6.8 to 6.10 and 6.16 may seem unnatural in view of the chosen universal 
classes $\mathscr{A}$. However, every example can easily be "blown up" to bigger universal classes $\mathscr{B}(\supseteq \mathscr{A})$ by considering in $\mathscr{B}$ the class $\mathscr{K}^{\prime}:=\mathscr{M} \cup(\mathscr{B} \backslash \mathscr{A})$. A straightforward but somewhat tedious checking shows that if $\mathscr{M}^{\prime}$ (respectively the corresponding $H$-radical $\rho^{\prime}$ ) has some property $(x)$ in $\mathscr{B}$, then $\mathscr{M}$ (respectively the corresponding $H$-radical $\rho$ ) has $(x)$ in $\mathscr{A}$ where $(x)$ stand for any property appearing in our scheme. Thus, no new properties can be added by this way of blowing up an example. For other types of blowing up, this is not the case (compare Examples 6.8 and 6.9). By the indicated method, some properties may disappear, but a lot of properties $(x)$ remain invariant under blowing up. For convenience, we give the complete list of these $(x)$ : (2'), closed under essential extentions, CE $\mathscr{M}$ E-class, $\mathscr{M}_{k} \subseteq \overline{\mathscr{M}}, \mathscr{U} \mathscr{M}=\mathscr{U} \mathscr{M}_{k}$, $\mathscr{U} \mathscr{M} \cap \mathscr{U} \mathscr{M}_{k}=0,\left(1^{\prime}\right), r$-hereditary, $\mathscr{R}_{p}$ hereditary, $\rho$ idempotent, (c), (E'), $\rho N \subseteq \mathscr{R}_{\rho}(N), \rho$ hereditarily idempotent on $\mathscr{S} \rho$.

\section{Acknowledgements}

This research was carried out while the second author visited the first author at the Technical University in Vienna. They are kindly thanked for their hospitality and the use of their facilities. The authors are indebted to Professor W. G. Leavitt for providing several or our examples.

\section{References}

[1] T. Anderson, K. Kaarli, and R. Wiegandt, 'Radicals and subdirect decompositions,' Comm. Algebra 13 (1985), 479-494.

[2] V. A. Andrunakievič, 'Radicals of associative rings, I,' Mat Sb. 44 (1958), 179-212 (in Russian; English transl. in Amer. Math. Soc. Transl. 52 (1966), 95-128).

[3] A. Buys, 'Radicals of $\Omega$-groups', Contributions to general algebra 4, pp. 23-35 (HölderPichler-Tempsky, B. G. Teubner, Wien, Stuttgart, 1987).

[4] N. Divinsky, Rings and radicals, (Allen and Unwin, London, 1965).

[5] G. A. P. Heyman and C. Roos, 'Essential extensions in radical theory for rings', J. Austral. Math. Soc. 23 (1977), 340-347.

[6] H. J. Hoehnke, 'Radikale in allgemeinen Algebren', Math. Nachr. 32 (1966), 347-383.

[7] K. Kaarli, 'Classification of irreducible $R$-groups over a semi-primary near-ring', Tartu Riikl. Al. Toimetised 556 (1981), 47-63 (in Russian).

[8] J. Krempa, 'Lower radical properties for alternative rings', Bull. Acad. Polon. Sci. Sér. Sci. Math. 23 (1975), 139-142.

[9] W. G. Leavitt, 'A minimally embeddable ring', Period. Math. Hungar. 12 (1981), 129-140.

[10] W. G. Leavitt, 'Hereditary upper radicals', Studia Sci. Math. Hungar. 16 (1981), 15-23.

[11] W. G. Leavitt, 'Upper radicals of regular classes', Acta Math. Acad. Sci. Hungar. 42 (1983), 213-220.

[12] W. G. Leavitt, (Private communication). 
[13] W. G. Leavitt and E. P. Armendariz, 'Non-hereditary semisimple classes', Proc. Amer. Math. Soc. 18 (1967), 1114-1117.

[14] W. G. Leavitt and L. C. A Van Leeuwen, 'Hereditariness of upper radical', Contributions to general algebra 4, 79-84 (Hölder-Pichler-Tempsky, B. G. Teubner, Wien, Stuttgart, 1987).

[15] R. Mlitz, 'Radicals and semisimple classes of $\Omega$-groups', Proc. Edinburgh Math. Soc. 23 (1980), 37-41.

[16] R. Mlitz and A. Oswald, 'Hypersolvable and supernilpotent radicals of near-rings', Studia Math., to appear.

[17] M. A. Rashid and R. Weigandt, 'The hereditariness of the upper radical', Acta Math. Acad. Sci. Hungar. 24 (1973), 343-347.

[18] Ju M. Rjabuhin, 'Radicals in $\Omega$-groups, II, Ideal-hereditary radicals', Mat. Issled. 3 vyp 4 (10) (1968), 108-135 (in Russian).

[19] Ju M. Rjabuhin, On supernilpotent and special radicals, Issled Alg. i Mat. Anal. 65-72, Akad. Nauk Moldov. SSR, Kishinew, 1965 (in Russian).

[20] Ju M. Rjabuhin, 'Lower radicals of rings', Mat. Zametki 2 (1967), 239-244 (in Russian).

[21] R. L. Tangeman and D. Kreiling, 'Lower radicals in non-associative rings', J. Austral. Math. Soc. 24 (1972), 419-423.

[22] S. Veldsman, 'Supernilpotent radicals of near-rings', Comm. Algebra 15 (1987), 2497-2509.

[23] R. Weigandt, Radica and semisimple classes of rings, (Queen's papers in pure and applied math., no. 37, Kingston, Ontario, 1974).

Technische Universität Wien

Institut für Angewandte und

Numerische Mathematik

A-1040 Wien

Austria
Department of Mathematics University of Port Elizabeth

P.O. Box 1600

6000 Port Elizabeth

South Africa 